\title{
Alginate Hydrogels as Injectable Drug Delivery Vehicles for Optic Neuropathy Treatment
}

\section{Courtney J. Maxwell}

The Ohio State University

\section{Andrew M. Soltisz}

The Ohio State University

\section{Wade W. Rich}

The Ohio State University

\section{Andrew Choi}

The Ohio State University

\section{Matthew A. Reilly}

The Ohio State University

Katelyn Swindle-Reilly ( $\square$ reilly.198@osu.edu )

The Ohio State University https://orcid.org/0000-0003-1739-0263

\section{Research Article}

Keywords: Optic neuropathy, ocular drug delivery, reactive oxygen species, methylene blue, hydrogel, alginate, calcium carbonate, controlled release

Posted Date: April 13th, 2021

DOl: https://doi.org/10.21203/rs.3.rs-391900/v1

License: (c) (1) This work is licensed under a Creative Commons Attribution 4.0 International License. 


\title{
Alginate Hydrogels as Injectable Drug Delivery Vehicles for Optic Neuropathy Treatment
}

Courtney J. Maxwell ${ }^{1}$, Andrew M. Soltisz ${ }^{1}$, Wade W. Rich ${ }^{1}$, Andrew Choi ${ }^{1}$, Matthew A. Reilly ${ }^{1,2}$, Katelyn E. Swindle-Reilly ${ }^{1,2,3}$

${ }^{1}$ Department of Biomedical Engineering, The Ohio State University, Columbus, OH, United States,

${ }^{2}$ William G. Lowrie Department of Chemical and Biomolecular Engineering, The Ohio State University, Columbus, OH, United States, ${ }^{3}$ Department of Ophthalmology and Visual Science, The Ohio State University, Columbus, OH, United States

\begin{abstract}
Optic neuropathy is the loss of visual acuity following damage to the optic nerve (ON). Traumatic optic neuropathy (TON) occurs when the optic nerve is injured following blunt or penetrating trauma to either the head or eye. Current management options for TON include the systemic delivery of corticosteroids and surgical decompression of the optic nerve; however, neither option alleviates the generation of reactive oxygen species (ROS) which are responsible for downstream damage to the ON. Addressing this limitation, an injectable alginate hydrogel system was developed to act as a drug delivery vehicle for methylene blue (MB), a confirmed ROS scavenger and neuroprotective agent. This MB-loaded polymeric scaffold has the ability to be injected as a liquid and rapidly form a gel around the optic nerve following the primary injury, allowing for the prolonged release of MB. The MB-loaded alginate hydrogels demonstrated minimal cytotoxicity to human retinal pigment epithelial (ARPE-19) cells and facilitated gradual MB release over 12 days. Additionally, the MB concentrations displayed a high degree of ROS scavenging after release from the alginate hydrogels, suggesting our approach may be successful in reducing ROS levels following ON injury.
\end{abstract}

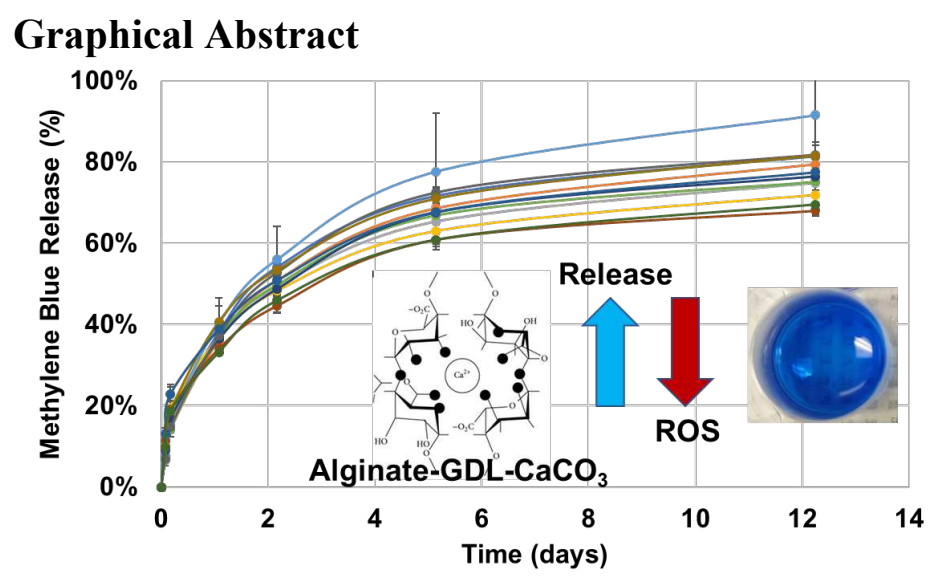




\section{Keywords}

Optic neuropathy; ocular drug delivery; reactive oxygen species; methylene blue; hydrogel; alginate; calcium carbonate; controlled release

\section{Introduction}

Optic neuropathy results in visual dysfunction due to optic nerve damage (e.g. glaucoma) and can be caused by several mechanisms. Traumatic optic neuropathy (TON) is an ocular injury in which the force or motion of the globe or orbital tissues is transferred from the eye or skull to the optic nerve $(\mathrm{ON})$. It can be characterized by transient or permanent vision impairment, associated vascular and edema damage and subsequent $\mathrm{ON}$ atrophy [1]. In civilians, $0.4 \%$ of trauma patients incur TON; this increases to 5\% in cases involving closed-head injuries [3]. Among soldiers, its incidence is higher, with ocular trauma representing up to $13 \%$ of recent battlefield injuries [4]. The current management options for the treatment of TON include the systemic delivery of corticosteroids and surgical decompression of the nerve. However, both treatments are ineffective at improving visual recovery, have side effects such as optic atrophy, complications following surgical decompression [5], and do not address secondary injury mechanisms such as the unattenuated generation of reactive oxygen species (ROS) [6]. Prolonged exposure to high ROS levels in the $\mathrm{ON}$ can cause a multitude of cellular dysregulations, namely the migration of inflammatory cells to the site of injury. These secondary injuries contribute to the formation of glial scarring [7], preventing tissue recovery by inhibiting signal transduction following the primary injury and contributing to permanent vision loss. Therefore, there is a demonstrated need to develop an effective treatment to sustain the release of ROS scavengers, thereby addressing the injuries caused by secondary injuries following TON.

Methylene blue (MB) is a potent ROS scavenger and neuroprotective agent capable of crossing the blood-brain barrier with a demonstrated inhibitory effect on glial cell migration in vivo [8 12]. These qualities, along with recent discoveries of recovery following traumatic brain injury (TBI) and stroke in rats $[13,14]$, makes MB a promising therapeutic candidate for TON treatment. Due to its small molecular weight and high-water solubility, direct local injection of MB to the ON would be inadequate, leading to rapid diffusion of MB away from the site of injury. By surrounding the injured nerve with a MB-loaded degradable scaffold, drug elution would be better facilitated, allowing for a more sustained therapeutic release. 
Alginate-based hydrogels were selected as the drug delivery vehicle in our study due to their biocompatible nature and extensive usage in tissue engineering and drug delivery applications $[15$, 16]. Typically, aqueous alginate is externally crosslinked by the addition of dissolved $\mathrm{Ca}^{2+}$ ions, forming a hydrated scaffold. This method was initially investigated for our TON treatment, but it induced rapid gelation, limiting its injection feasibility through a small gauge needle. Draget et al. [16], introduced an internal method of alginate crosslinking (Figure I), in which insoluble calcium carbonate $\left(\mathrm{CaCO}_{3}\right)$ particles are evenly distributed throughout the alginate solution before the addition of a slow hydrolyzing proton donor, in our case, D-glucono-lactone (GDL) [17]. The internal method of crosslinking slows the process of gelation to a rate that accommodates mixing of all components, subsequent injection through a small gauge needle and in situ hydrogel formation. By modulating the concentrations of the hydrogel's constituents, we developed a hydrated polymeric scaffold with tunable capabilities. Previous studies have evaluated alginate gels crosslinked with calcium carbonate as an injectable vehicle for osteoblast delivery in tissue engineering applications [19 - 21]; however, an extensive evaluation of its drug delivery capabilities has yet to be studied. Given the need for a TON treatment that addresses the current limitations, internally crosslinked MB-loaded injectable alginate hydrogels could potentially lower the concentration of ROS and effectively improve visual recovery following injury.

With the above considerations, the purpose of this study was to synthesize alginate hydrogels loaded with MB through internal crosslinking and in situ gelation. We hypothesized that MB stimulated by oxidative stress could achieve ROS scavenging and effectively halt the generation of deleterious reactive species. 


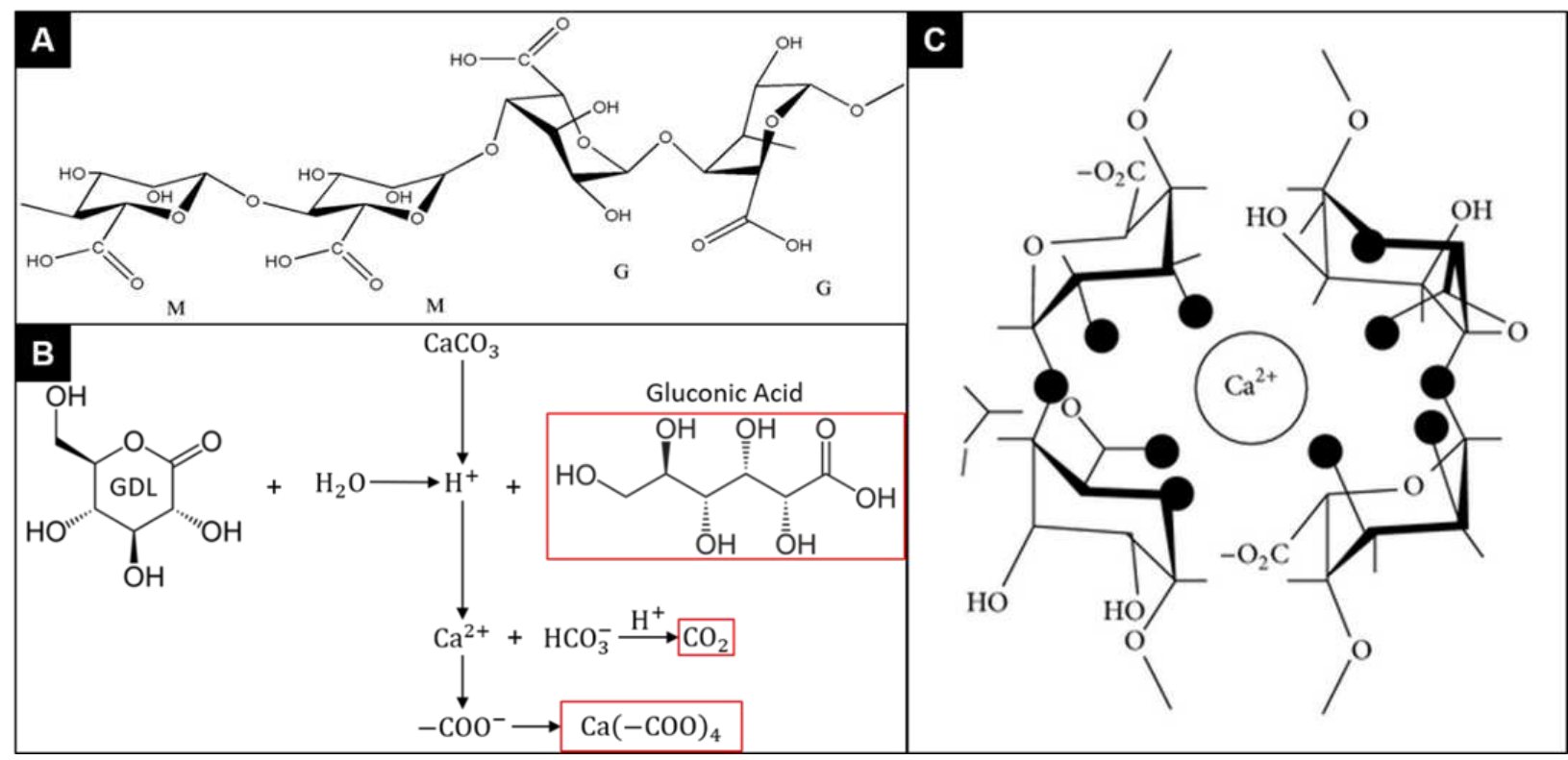

Figure I. Diagram of crosslinking reaction and final hydrogel structure. [22] (A) Alginate is a polysaccharide copolymer composed of two residues, (1-4)-linked $\beta$-D mannuronate $(\mathrm{M})$, and $\alpha$-L-guluronate $(\mathrm{G})$. The patterning and ratio of these residues can significantly impact the material properties of hydrogels. (B) Schematic of the crosslinking reaction between the proton donor D-glucono-lactone (GDL), the calcium ion source $\mathrm{CaCO}_{3}$ and the alginate polymer. The reaction generates three products - gluconic acid, carbon dioxide, and the calcium ion-alginate complex. (C) [18] Once $\mathrm{Ca}^{2+}$ is freed by GDL, the free ion interacts with alginate's carboxyl group to from ionic crosslinking between polymers.

\section{Materials and Methods}

\subsection{Materials}

Sodium alginate (Protanal PH 1033) was provided by FMC Biopolymer (Philadelphia, Pennsylvania). Methylene blue (MB) and Dulbecco's phosphate-buffered saline (DPBS) were purchased from Sigma-Aldrich (Saint Louis, Missouri). Calcium carbonate $\left(\mathrm{CaCO}_{3}\right)$ was purchased from ChemProducts (Tualatin, Oregon). D-(+)-glucono-1,5-lactone (GDL) was purchased from Alfa Aesar (Haverhill, Massachusetts). Colorimetric 3-(4,5-dimethylthiazol-2-yl)5-(3-carboxymethoxyphenyl)-2-(4-sulfophenyl)-2H-tetrazolium (MTS) assay and 2,7dichlorodihydrofluorescein diacetate (DCFH-DA) assay were purchased from Fisher Scientific Inc. (Hampton, NH). Human retinal pigment endothelial cells (ARPE-19, ATCC CRL-2302) were purchased from American Type Culture Collection (ATCC) (Manassas, VA). Dulbecco's Modified Eagle's/Nutrient Mixture F-12 Ham's Medium (DMEM/F-12), phenol-free DMEM, fetal calf serum (FCS), penicillin-streptomycin (PS), trypsin, dimethyl sulfoxide (DMSO) and hydrogen peroxide $\left(\mathrm{H}_{2} \mathrm{O}_{2}\right)$ were all purchased from Thermo Fisher Scientific (Waltham, MA, USA). 


\subsection{Hydrogel Synthesis}

Sodium alginate hydrogels were synthesized based on methods reported in literature with modifications [17]. 21 total hydrogel formulations were prepared and evaluated using design of experiments to modify alginate, $\mathrm{CaCO}_{3}$ and GDL concentrations. The first 9 formulations were selected based on their consistency in forming solid homogeneous hydrogels (Table I). They served as the models for $\mathrm{pH}$ testing in preliminary experiments (see Section 2.3).

Briefly, sodium alginate $\left(0.63 \%-1.85 \%\right.$ final $\mathrm{w} / \mathrm{v}$ in gel) was dissolved in $\mathrm{DI}_{2} \mathrm{H}_{2} \mathrm{O}$ by vortexing for 30 seconds and heating in $37^{\circ} \mathrm{C}$ water for 24 hours. Aqueous $1 \mathrm{mg} / \mathrm{mL} \mathrm{MB}$ was added to a final concentration of $0.05 \mathrm{mg} / \mathrm{mL}$, followed by the addition of $\mathrm{CaCO}_{3}$ and vortexed. As gelation is initiated rapidly following addition of GDL, the solution was quickly transferred to a mold or onto the rheometer stage following subsequent mixing of all components.

To prepare the remaining hydrogel formulations $(10-21), \mathrm{GDL}: \mathrm{CaCO}_{3}$ molar ratio concentrations were based off of the original 9 formulations that exhibited a neutral $\mathrm{pH}$ of $7.0 \pm 1.0$ (Figure II, Table I). These GDL: $\mathrm{CaCO}_{3}$ ratios ranged from $0.125-1.00$. The $\mathrm{Ca}^{2+}$ :alginate monomer molar concentrations were also evaluated as a factor and varied from $0.5-1.5$. The ratios were selected to assess the influence of alginate and crosslinker concentrations on drug release, cytotoxicity and viscoelastic properties. Hydrogel formulations 10, 16 and 21 were selected as low, medium and high concentration hydrogels due to their GDL: $\mathrm{CaCO}_{3}$ molar ratios; $0.125,0.500$ and 1.00, respectively. They were analyzed further in cytotoxicity and ROS experimentation (see sections $2.4,2.5)$.

Table I. Composition of hydrogel formulations prepared and evaluated. The alginate hydrogels were designed based on their ability to form solid homogenous hydrogels. Formulations consist of $180 \mathrm{mg}$ sodium alginate with varying molar concentrations of $\mathrm{CaCO}_{3}$ and GDL. The final GDL and $\mathrm{CaCO}_{3}$ concentrations were modulated based on preliminary hydrogels $(1-9)$. Molar concentrations of GDL:CaCO $\mathrm{C}_{3}$ ratios ranged from 0.125 - 1.00. Aqueous 1 $\mathrm{mg} / \mathrm{mL} \mathrm{MB}$ was also added to each formulation., except in ROS studies in which MB ranged from $0.05-2.0 \mathrm{mg} / \mathrm{mL}$ to access the influence of MB concentration on ROS scavenging ability.

\begin{tabular}{ccccc}
\hline $\begin{array}{c}\text { Formulation } \\
\text { ID }\end{array}$ & $\begin{array}{c}\mathbf{C a}^{2+}: \text { Alginate Monomer } \\
(\mathbf{m o l}: \mathbf{m o l})\end{array}$ & $\begin{array}{c}\text { GDL:CaCO3} \\
(\mathbf{m o l}: \mathbf{m o l})\end{array}$ & $\begin{array}{c}{[\mathrm{CaCO}]} \\
(\mathbf{g} / \mathbf{L})\end{array}$ & $\begin{array}{c}{[\mathrm{GDL}]} \\
(\mathrm{g} / \mathrm{L})\end{array}$ \\
\hline 1 & 0.469 & 2.493 & 1.600 & 7.100 \\
2 & 0.733 & 1.596 & 2.500 & 7.100 \\
3 & 0.997 & 1.173 & 3.400 & 7.100 \\
4 & 0.469 & 3.301 & 1.600 & 9.400
\end{tabular}




\begin{tabular}{|c|c|c|c|c|}
\hline 5 & 0.733 & 2.113 & 2.500 & 9.400 \\
\hline 6 & 0.997 & 1.553 & 3.400 & 9.400 \\
\hline 7 & 0.469 & 4.109 & 1.600 & 11.700 \\
\hline 8 & 0.733 & 2.630 & 2.500 & 11.700 \\
\hline 9 & 0.997 & 1.933 & 3.400 & 11.700 \\
\hline 10 & 0.500 & 0.125 & 0.400 & 0.400 \\
\hline 11 & 0.500 & 0.250 & 0.900 & 0.800 \\
\hline 12 & 0.500 & 0.500 & 1.700 & 1.500 \\
\hline 13 & 0.500 & 1.000 & 3.400 & 3.000 \\
\hline 14 & 1.000 & 0.125 & 0.400 & 0.800 \\
\hline 15 & 1.000 & 0.250 & 0.900 & 1.500 \\
\hline 16 & 1.000 & 0.500 & 1.700 & 3.000 \\
\hline 17 & 1.000 & 1.000 & 3.400 & 6.100 \\
\hline 18 & 1.500 & 0.125 & 0.400 & 1.100 \\
\hline 19 & 1.500 & 0.250 & 0.900 & 2.300 \\
\hline 20 & 1.500 & 0.500 & 1.700 & 4.600 \\
\hline 21 & 1.500 & 1.000 & 3.400 & 9.100 \\
\hline
\end{tabular}

\subsection{Hydrogel $\mathrm{pH}$}

The $\mathrm{pH}$ values of alginate hydrogel formulations $1-9$ were evaluated using a calibrated $\mathrm{pH}$ probe (Mettler Toledo, InLab Expert Pro-ISM, Columbus, $\mathrm{OH}$ ) for 72 hours to evaluate $\mathrm{pH}$ evolution and determine the final compositions of hydrogel formulations $10-21$. The final $\mathrm{pH}$ was reported as the equilibrium $\mathrm{pH}(\mathrm{pH})$.

\subsection{Hydrogel Cytotoxicity}

The biocompatibility of representative low, medium and high concentration hydrogels (formulations 10, 16, 21) were evaluated using the MTS assay and adapted from the methods of Niu et al. [24]. ARPE-19 cells were first seeded at $5 \times 10^{3}$ cells per well in a 96 well-plate and incubated for 24 hours in $200 \mu \mathrm{L}$ base media (DMEM/F12, 10\% FBS, 1\% PS). $1 \mathrm{~mL}$ hydrogels were formed in $15 \mathrm{~mL}$ conical tubes and allowed to completely gel for 72 hours before 60 - minute UV light exposure, ensuring sterility [25]. The hydrogels were then immersed in $1 \mathrm{~mL}$ base media for 24 hours before media collection. The cells were incubated in $200 \mu \mathrm{L}$ samples for 48 hours 
prior to performing the MTS assay. A positive control of base media, negative control of 1:9 dimethyl sulfoxide (DMSO): growth media [26] and blank of phenol-free DMEM were used to validate the assay. After incubation, the hydrogel-soaked media was removed and each well washed three times with $200 \mu \mathrm{L}$ DPBS. Following, $180 \mu \mathrm{L}$ of phenol-free growth media and 20 $\mu \mathrm{L}$ MTS reagent was added to each well and allowed to incubate for 1 hour. Optical density (OD) of the MTS-treated media was measured at $490 \mathrm{~nm}$ using a BioTek Elx808 plate reader (Winooski, VT).

\subsection{ROS Scavenging}

A DCFH-DA assay was used to evaluate the ability of MB to scavenge ROS in cell culture based on the methods of Ludmila et al. (2005) [27]. ARPE-19 cells were seeded on a 96 well-plate at a density of $2 \times 10^{4}$ cells per well in DMEM/F12 media supplemented with 10\% FBS and 1\% PS

and incubated for 24 hours. $\mathrm{MB}$ at concentrations of $0 \mathrm{mg} / \mathrm{L}$ (positive control), $0.05 \mathrm{mg} / \mathrm{L}, 0.25$ $\mathrm{mg} / \mathrm{L}, 0.50 \mathrm{mg} / \mathrm{L}, 1.0 \mathrm{mg} / \mathrm{L}$ and $2.0 \mathrm{mg} / \mathrm{L}$ and a positive control of $\mathrm{H}_{2} \mathrm{O}_{2}$ and a negative control of DPBS were added to the wells and incubated for 24 hours. Following incubation, the media was removed and $100 \mu \mathrm{L}$ of DCFH-DA solution was added to each well and incubated for $1-2$ hours. The cells were washed with DPBS once and the excitation and emission wavelengths; $485 \mathrm{~nm}$ and $535 \mathrm{~nm}$, respectively, were measured using a microplate reader.

To further confirm the ROS scavenging ability of MB, $1 \mathrm{~mL}$ hydrogels (formulations $10,16,21$ ) were formed in $15 \mathrm{~mL}$ conical tubes and allowed to gel for 72 hours. Following gelation, the hydrogels were exposed to UV light for one hour to sterilize. The hydrogels were then immersed in $1 \mathrm{~mL}$ base media for 24 hours before media collection. ARPE-19 cells were seeded on a 96 well-plate with $2 \times 10^{4}$ cells per well in base growth media and allowed to grow for 24 hours. The culture media was removed and the hydrogel - soaked medium was added to the wells. Hydrogen peroxide (10 $\mu \mathrm{L}, 600 \mu \mathrm{M}$ final concentration) was added to test wells while DPBS was added to the other wells as a negative control. Additionally, hydrogel formulation 16 (medium concentration hydrogel) without MB was included as a negative control. Cells were incubated for 24 hours. Following incubation, the media was removed and $100 \mu \mathrm{L}$ of DCFH-DA solution was added to each well and incubated for $1-2$ hours. The cells were thoroughly washed with PBS, and the excitation and emission wavelengths were measured at $485 \mathrm{~nm}$ and $535 \mathrm{~nm}$, respectively. 


\subsection{Gelation Kinetics and Mechanical Properties}

Oscillatory shear rheology was used to characterize the gelation kinetics, strain amplitude response, and frequency response of alginate hydrogel formulations $10-21$ [22]. The rheometer used was a Malvern Panalytical Kinexus Ultra+ (Malvern, United Kingdom) with a $20 \mathrm{~mm}$ titanium parallel plate upper geometry (PU20 SW1511 TI) and aluminum lower geometry (PLC61 S3722 AL). For all rheological tests, the gap height between the lower and upper geometries, the temperature and sample size were kept constant at $1 \mathrm{~mm}, 37^{\circ} \mathrm{C}$ and $375 \mu \mathrm{L}$, respectively.

To measure the gelation kinetics of alginate hydrogels, the alginate solution was dispensed as a liquid directly onto the lower geometry of the rheometer immediately following the addition and mixing of GDL. A constant frequency and strain amplitude of $1 \mathrm{~Hz}$ and $1 \%$ respectively (within linear viscoelastic region), were applied to the sample with its resulting shear stress measured every 5 seconds for 2 hours. The gelation time was defined as the time which gelation had terminated and was determined from the constant frequency and strain test as the first timepoint where complex shear modulus $\left(\mathrm{G}^{*}\right)$ did not increase by more than $1 \%$ of the average of the 10 previously collected measurements [22]. A frequency sweep test immediately followed the gelation test, evaluating the frequency response of the hydrogel. Here, a constant strain amplitude of $1 \%$ was applied to the sample while frequency increased from $1 \mathrm{~Hz}$ to $100 \mathrm{~Hz}$. The stiffness of the hydrogels is reported as the value of $\mathrm{G}^{*}$ at $1 \mathrm{~Hz}$ from frequency sweep tests.

Representative low, medium, and high concentration $\mathrm{CaCO}_{3}$ and GDL hydrogels (formulations 10,16 , and 21 , respectively) were additionally subjected to an amplitude sweep test to evaluate strain amplitude response. A constant frequency of $1 \mathrm{~Hz}$ was applied to the sample while the strain amplitude increased from $0.1 \%$ to $100 \%$, and resulting stress was measured.

\subsection{Hydrogel Swelling and MB Release}

$1 \mathrm{~mL}$ samples of hydrogel formulations $10-21$ were cast in pre-weighed $15 \mathrm{~mL}$ conical tubes and weighed. Formulations $1-9$ were not included as preliminary studies determined only GDL: $\mathrm{CaCO}_{3}$ ratios of $0.25-1.0$ were relevant for our studies due to the determined crosslinking maximum between $\mathrm{Ca}^{2+}$ ions and alginate.

Hydrogels were then immersed in $1 \mathrm{~mL}$ DPBS modified without calcium chloride $\left(\mathrm{CaCl}_{2}\right)$ and magnesium chloride $\left(\mathrm{MgCl}_{2}\right)$ at $37^{\circ} \mathrm{C}$ and at regular intervals $(0,1,3,7$, and 14 days), DPBS was 
removed and the mass of the hydrogels was recorded. Results were calculated according to the following equation:

$$
Q=\frac{M_{S}-M_{D}}{M_{D}} \times 100 \%
$$

Here, $\mathrm{Q}$ is the swelling ratio, $\mathrm{M}_{\mathrm{s}}$ is the mass of the formed hydrogel following incubation in DPBS at $37^{\circ} \mathrm{C}$ and excess water removal and $\mathrm{M}_{\mathrm{D}}$ is the mass of the $1 \mathrm{~mL}$ alginate solution placed in the tube [23].

The release kinetics of MB were evaluated using the same formulations $(10-21)$ evaluated for swelling. $1 \mathrm{~mL}$ hydrogels $(10-21)$ loaded with $1 \mathrm{mg} / \mathrm{mL} \mathrm{MB}$ were created. Following immersion in DPBS and incubation at $37^{\circ} \mathrm{C}, 1 \mathrm{~mL}$ DPBS was removed at the given intervals $(0,1,3,7$, and 14 days). $100 \mu \mathrm{L}$ samples of the DPBS were placed in a 96 well-plate and absorbance measured. The concentration of MB remaining in hydrogels following DPBS incubation was then determined using a standard concentration-absorbance curve measured at $630 \mathrm{~nm}$ using a plate reader (BioTekElx808).

\subsection{Hydrogel Degradation}

$1 \mathrm{~mL}$ hydrogel solutions based on formulations $10-21$ were cast in pre-weighed $15 \mathrm{~mL}$ conical tubes and weighed. After incubation at $37^{\circ} \mathrm{C}$ for 72 hours, excess water was removed from tube and hydrogels were weighed again to determine weight following incubation. Hydrogels were immersed in $10 \mathrm{~mL} 1 \mathrm{X}$ DPBS with $\mathrm{MgCl}_{2}$ and $\mathrm{CaCl}_{2}$ at $37^{\circ} \mathrm{C}$ for $0,1,3,7$ or 14 days. At each timepoint, the DPBS was removed, the hydrogels were frozen at $-80^{\circ} \mathrm{C}$ for 24 hours and lyophilized for 24 hours. Hydrogel degradation was reported as the percentage change in the mass

of dry components used to create the hydrogel to the dried hydrogel mass after freezing and lyophilization.

\subsection{Statistical Analysis}

Data analysis was performed using two-tailed student t-test. Statistical significance was defined as $\mathrm{p}<0.05$. All values and data points are reported as the average \pm standard deviation.

\section{Results}




\subsection{Hydrogel $\mathrm{pH}$}

The $\mathrm{pH}$ of hydrogel formulations $1-9$ was recorded for 72 hours (Figure IIA), with all formulations initially at $\sim 6 \mathrm{pH}$. Formulations demonstrated clear $\mathrm{pH}$ value groupings based on GDL: $\mathrm{CaCO}_{3}$ molar ratios. Formulations in the lower group $(1,4,5,7,8)$ had ratios greater than two, whereas the upper group $(2,3,6,9)$ had ratios less than two. The GDL: $\mathrm{CaCO}_{3}$ molar ratio was plotted against $\mathrm{pH}_{\mathrm{E}}$ over 72 hours (Figure IIB). Higher variability within the groupings is observed in the lower group with $\mathrm{pH}$ values ranging from $\sim 3.5$ to $\sim 4.5$; however, the variability may be explained by the larger range of GDL: $\mathrm{CaCO}_{3}$ ratios. Greater molar concentration of GDL and $\mathrm{CaCO}_{3}$ caused lower $\mathrm{pH}$. Therefore, formulations with close to neutral $\mathrm{pH}$ were evaluated in subsequent studies.
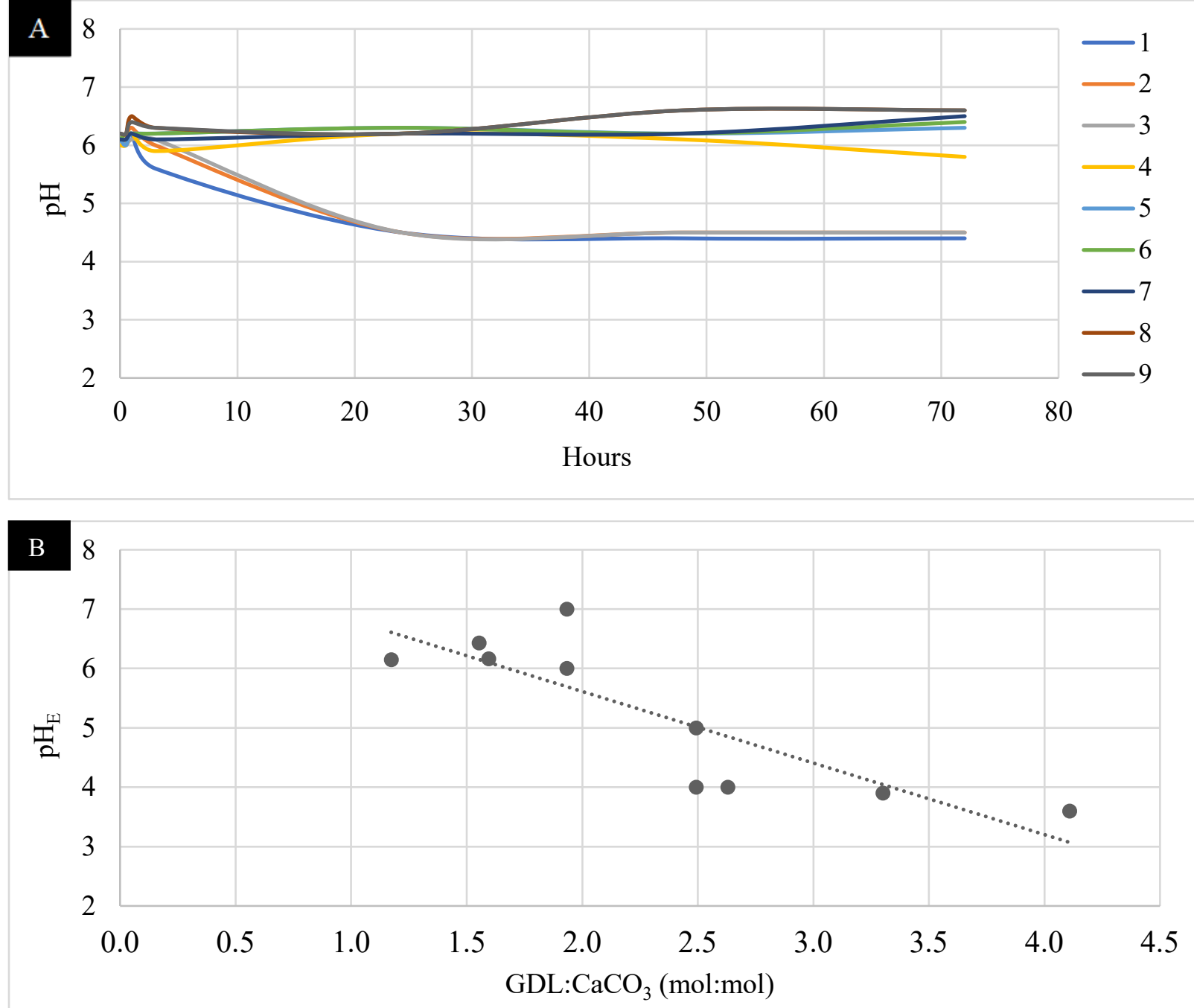

Figure II. Characterization of pH of hydrogel formulations 1 - 9. (A) Evolution of hydrogel pH over 72 hours. Formulations exhibit clear groupings of $\mathrm{pH}$ values. (B) Plot of hydrogel equilibrium $\mathrm{pH}\left(\mathrm{pH}_{\mathrm{E}}\right)$ reached after 72 hours of gelation. There is a linear and inverse relationship between GDL:CaCO 3 and $\mathrm{pH}_{\mathrm{E}}$ with an $\mathrm{R}^{2}$ of $0.8(\mathrm{p}<0.0001)$. 


\subsection{Rheological Characterization}

As shown in Table I, alginate hydrogels $10-21$ were prepared by varying $\mathrm{CaCO}_{3}$ and GDL concentrations. Time sweep rheology analysis (Figure III, Table II) found that different concentrations of the hydrogel components had an observable influence on complex shear modulus $\left(\mathrm{G}^{*}\right)$. As the concentrations of both $\mathrm{CaCO}_{3}$ and GDL increased, the complex shear modulus also increased. Increasing GDL content significantly increased $\mathrm{G}^{*}$ more than the addition of $\mathrm{CaCO}_{3}$. Additionally, complex modulus was dependent on GDL: $\mathrm{CaCO}_{3}$ ratios. Lower ratios corresponded lowered moduli and vice versa. All gelation times for formulations excluding 11 and 14 were significantly different from each other $(\mathrm{p}<0.05)$. Gelling time was found to be tunable, decreasing with higher concentrations of both $\mathrm{GDL}$ and $\mathrm{CaCO}_{3}$. All hydrogels exhibited a storage modulus significantly greater than their loss moduli and had a $\mathrm{G}^{*}$ of at least $35 \mathrm{~Pa}$ at $1 \mathrm{~Hz}$.

Table II. Quantification of the complex shear modulus and gelation times of hydrogels $10-21$. All hydrogels had a $\mathrm{G}^{*}$ of at least $35 \mathrm{~Pa}$ at $1 \mathrm{~Hz}$ and took at least 1 hour to reach equilibrium stiffness.

\begin{tabular}{ccc}
\hline Formulation & Gelation Time (s) & $\mathbf{G}^{*} \mathbf{1 ~ H z}(\mathbf{P a})$ \\
\hline 10 & $2803 \pm 40$ & $36 \pm 16$ \\
11 & $2025 \pm 120$ & $60 \pm 21$ \\
12 & $1223 \pm 91$ & $125 \pm 48$ \\
14 & $2270 \pm 26$ & $60 \pm 26$ \\
15 & $1517 \pm 99$ & $113 \pm 53$ \\
16 & $1275 \pm 248$ & $247 \pm 151$ \\
17 & $707 \pm 59$ & $559 \pm 34$ \\
18 & $2190 \pm 42$ & $70 \pm 34$ \\
19 & $1400 \pm 72$ & $187 \pm 92$ \\
20 & $1055 \pm 78$ & $312 \pm 134$ \\
21 & $660 \pm 198$ & $225 \pm 111$ \\
\hline
\end{tabular}




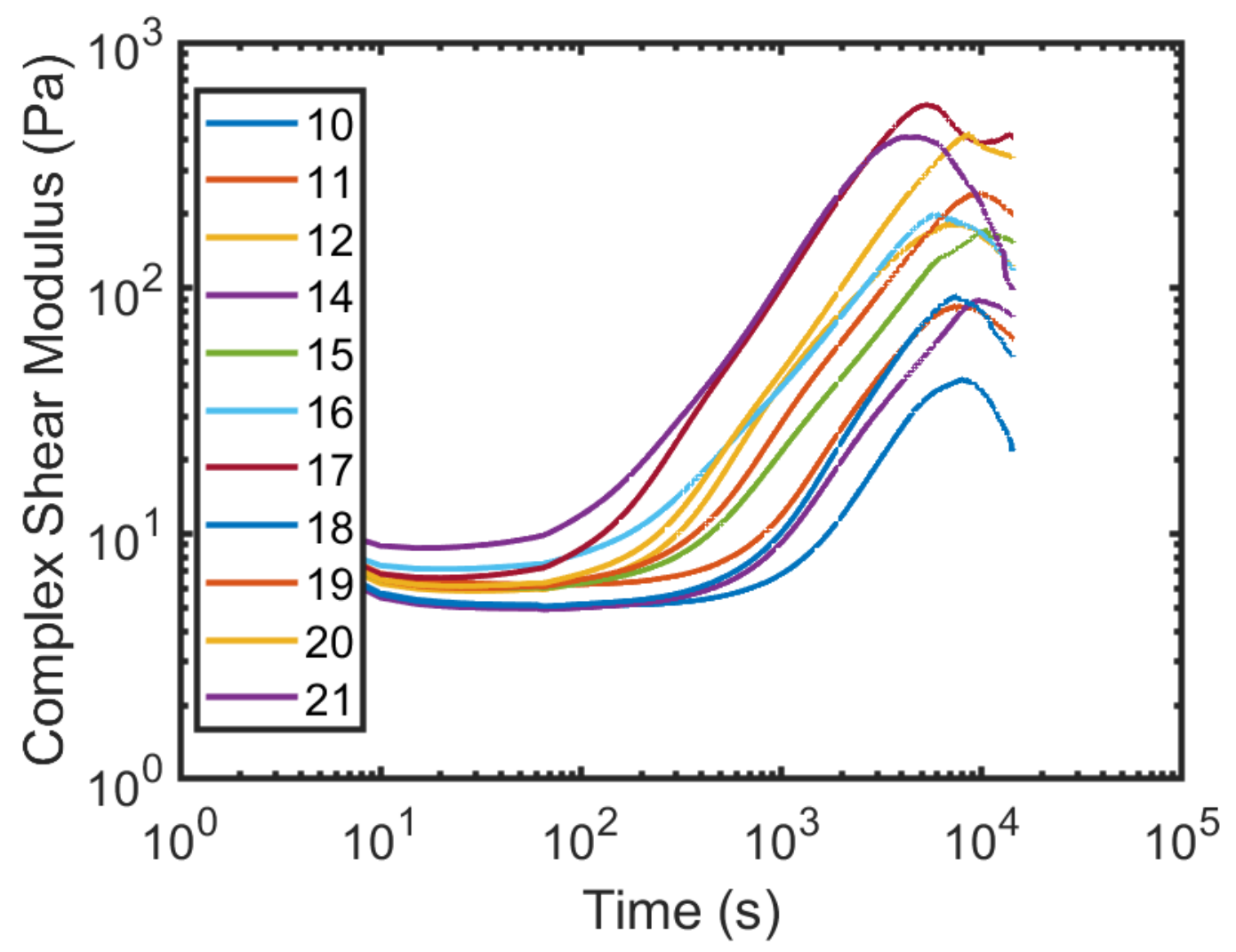

Figure III. Gelation characterization of hydrogel formulations. Time sweep results of hydrogel formulations $10-$ 21, excluding 13. Formulations had observable groupings of low and high GDL: $\mathrm{CaCO}_{3}$ ratio hydrogels. Gelation times ranged from $707 \pm 59$ to $2803 \pm 40$ seconds.

The observed influence of the hydrogel composition on $\mathrm{G}^{*}$ is detailed in Figure III. When the $\mathrm{Ca}^{2+}$ :alginate and GDL: $\mathrm{CaCO}_{3}$ were $0.500-1.000 \mathrm{~mol}: \mathrm{mol}$ and $0.125-0.250 \mathrm{~mol}: \mathrm{mol}$ respectively, $G^{*}$ gradually increased with time and their respective gelation times were among the lowest (formulations 10, 11, 12, 14, 15, 18), ranging from 1517-2803 seconds, or 20 - 48 minutes. When the $\mathrm{Ca}^{2+}$ :alginate and GDL:CaCO 3 were $1.000-1.500 \mathrm{~mol}: \mathrm{mol}$ and $0.250-0.500 \mathrm{~mol}: \mathrm{mol}$ respectively, $\mathrm{G}^{*}$ increased more rapidly with time and their respective gelation times ranged from 1055 - 1400 seconds i.e. 17 - 23 minutes (formulations 16, 19, 20). Lastly, when $\mathrm{Ca}^{2+}$ :alginate and GDL: $\mathrm{CaCO}_{3}$ mol concentrations were $0.500-1.000$ and $0.125-0.250 \mathrm{~mol}: \mathrm{mol}$ respectively, $\mathrm{G}^{*}$ dramatically increased within a short period of time and their respective gelation times were among the fastest, averaging around $660-707$ seconds or 11 minutes to gel completely (formulations 17, 21). 

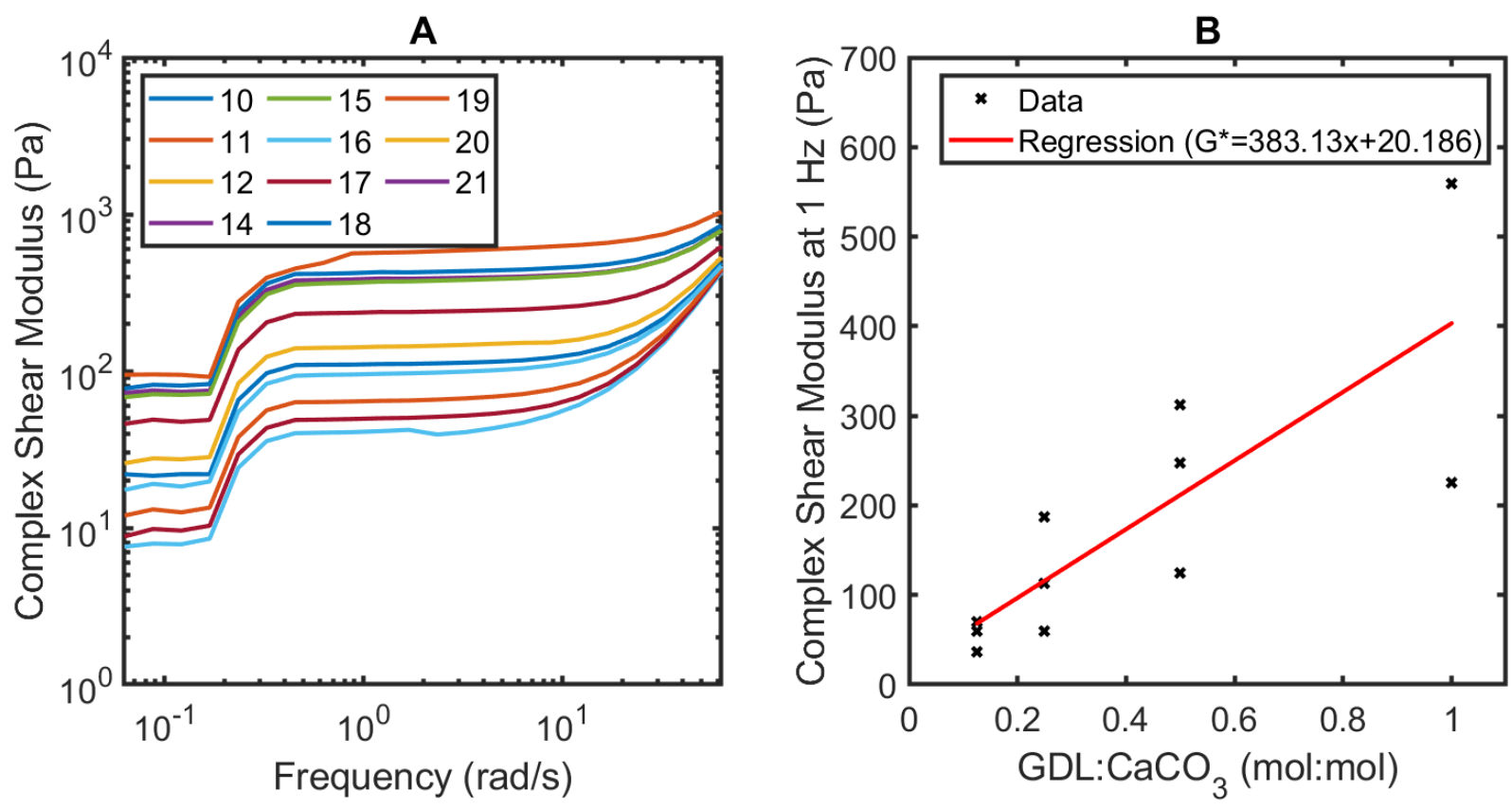

Figure IV. Frequency sweep results of hydrogel formulations. (A) Frequency sweep data from hydrogel formulations 10-21 (except 13). There is a positive exponential relationship between increasing frequency and complex shear stress. (B) Complex shear stress $\left(\mathrm{G}^{*}\right)$ as a function of concentration ratio $\left(\mathrm{CaCO}_{3}\right.$ :GDL) from hydrogel formulations $10-21$ at low frequencies. GDL: $\mathrm{CaCO}_{3}$ ratios significantly influence $\mathrm{G}^{*}$, with higher ratios contributing to high complex shear stresses $(\mathrm{p}<0.05)$.

Immediately following the gelation test, a frequency sweep was run on each hydrogel sample in triplicate with the result reported as the average \pm standard deviation $(n=3)$. Figure IV details the viscoelastic properties of the alginate hydrogels.

Similar to the grouping for the gelation test, there was grouping observed between low, medium and high concentration alginate hydrogels. The lower crosslinker concentration hydrogels (GDL: $\mathrm{CaCO}_{3}$ ratios of 0.125 and 0.250 ) corresponded with softer hydrogels and lower complex shear stress whereas higher concentration hydrogels (GDL:CaCO 3 ratios of 0.5 and 1.0) were stiffer and therefore had a higher complex shear stresses. The data show that by varying the components of the gels, a significant influence on complex shear modulus and gelation time were observed. 

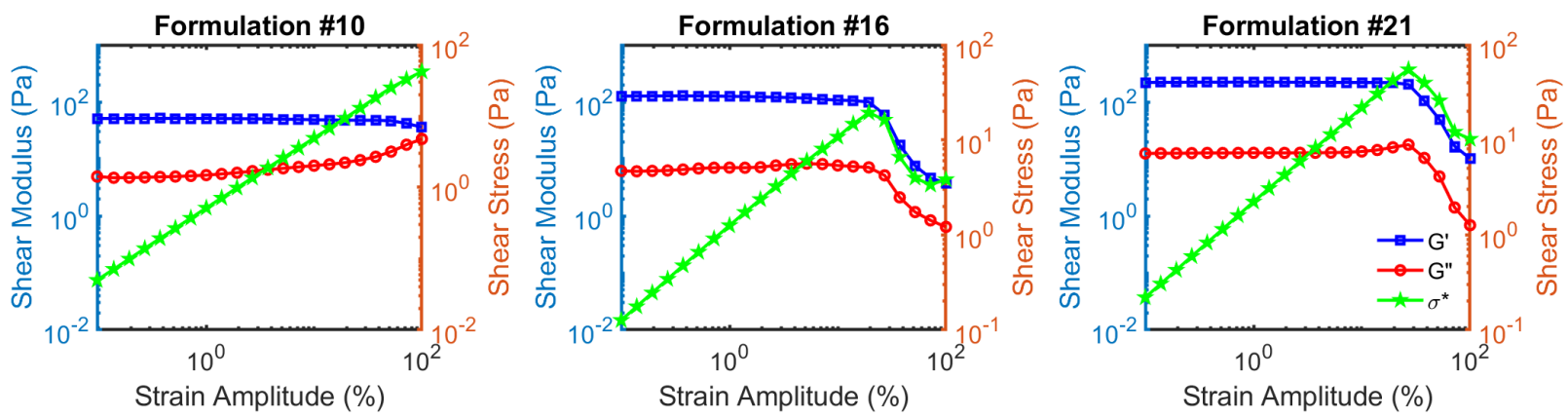

Figure V. Rheological characterization of hydrogel formulations. Amplitude sweep data from representative hydrogels. A linear viscoelastic region of stiffness response corresponding to $1 \mathrm{~Hz}$ dynamic shear is observed up to $1 \%$ strain.

The strain amplitude response of low, medium, and high concentration hydrogels of both $\mathrm{CaCO}_{3}$ and GDL $(10,16,21)$ were evaluated via amplitude sweep. Low (formulation 10) and high (formulation 21) concentration hydrogels and medium (16) and high concentration hydrogels were significantly different from each other ( $\mathrm{p}$ values 0.0021 and 0.0006 , respectively). All hydrogels contained a linear viscoelastic (LVE) region response to dynamic shear stress, originating at $\sim 1 \%$ strain and ending at $\sim 20 \%$ (Figure V). Maximum shear stress varied among the hydrogels. Formulation 21 demonstrates a sharp increase in complex shear strain from followed by a decrease around $20 \%$ complex shear strain, indicative of "fracturing."

\subsection{Swelling and MB Release of Alginate Hydrogels}

The swelling and MB release profile of the hydrogels was recorded in vitro over a period of 14 days (Figure VIB and VIIA, respectively). The degree of equilibrium swelling varied among hydrogels, ranging from $0-150 \%$. Formulations 12 and 21 had the lowest and highest swelling percentage, respectively, correlating to low and high GDL: $\mathrm{CaCO}_{3}$ ratios. The degree of swelling varied is indicative of the components within the hydrogels. Low to medium concentration (of both $\mathrm{CaCO}_{3}$ and GDL) hydrogels had degrees of swelling reported around $100-120 \%$, whereas high concentration hydrogels had swelling above $120 \%$. 


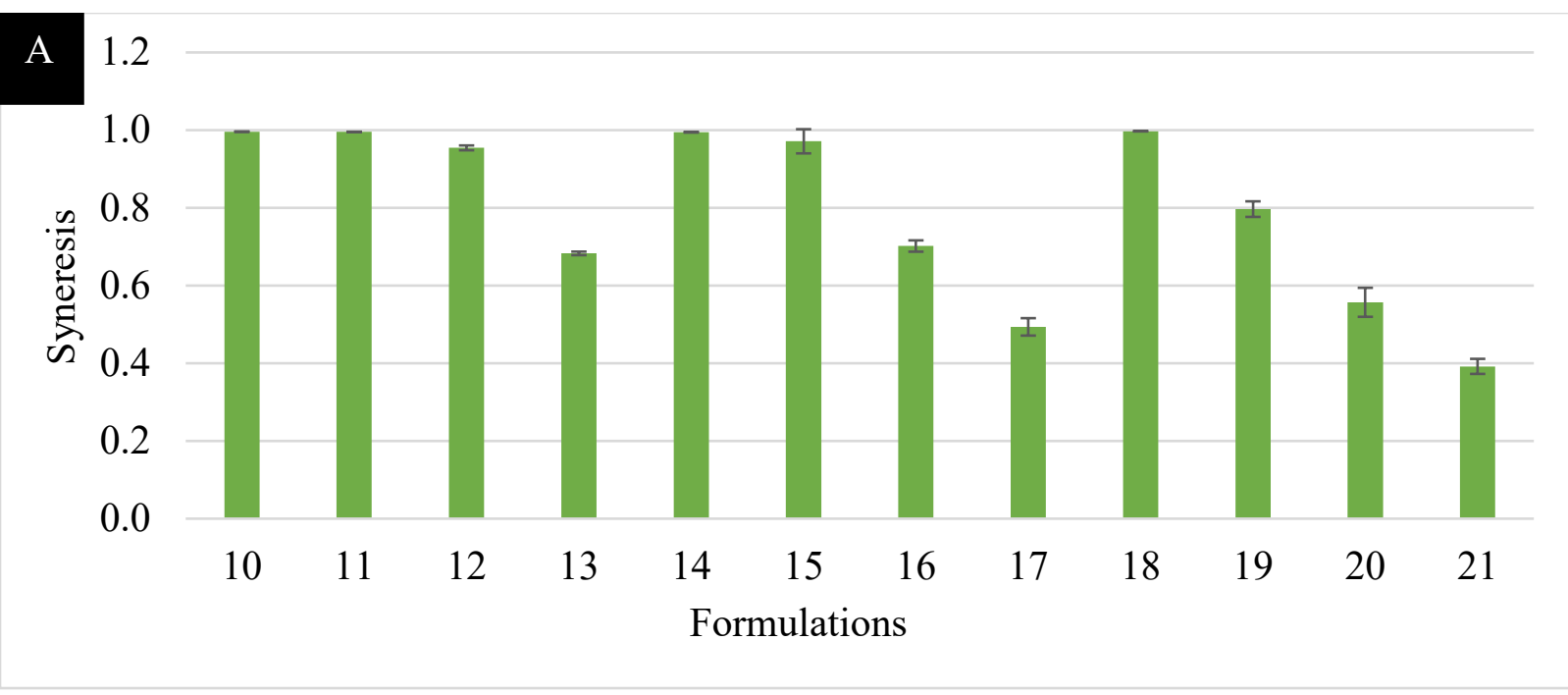

B

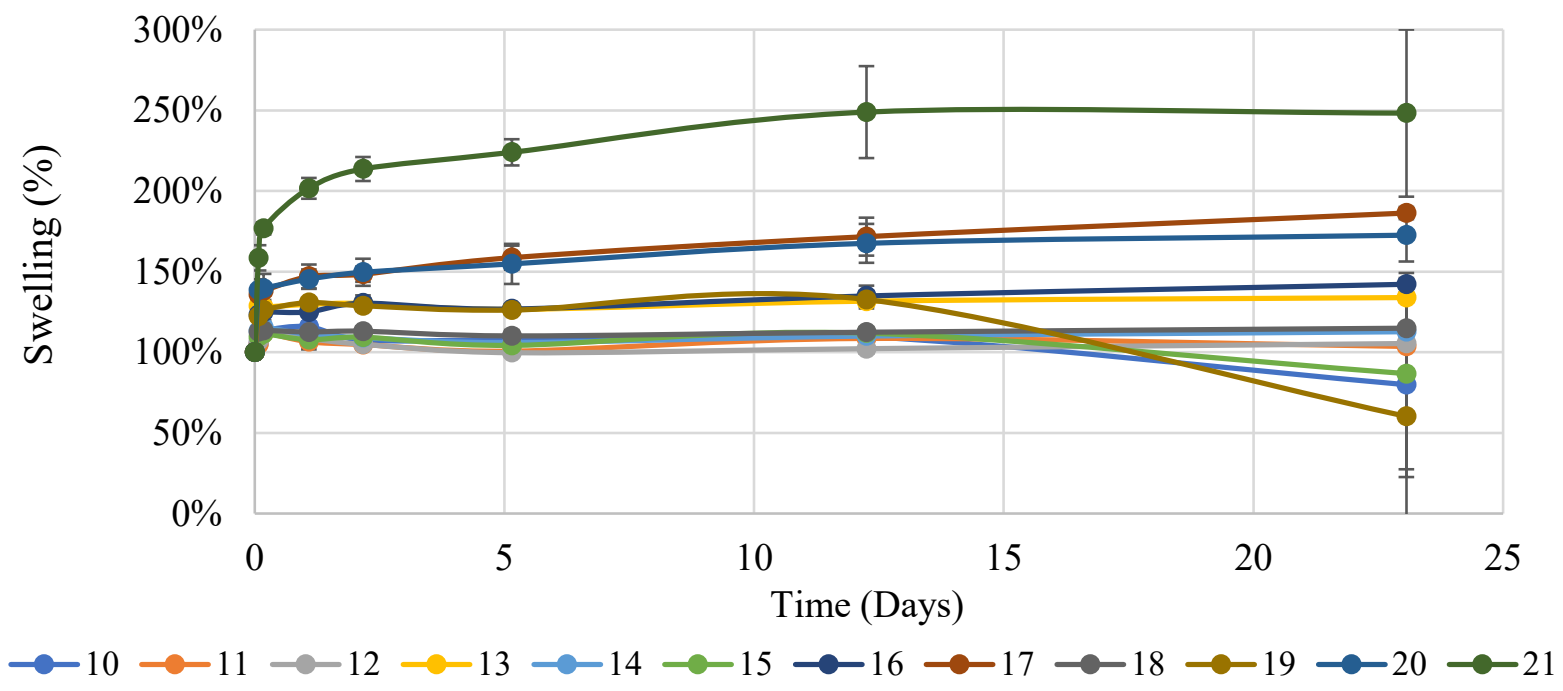

Figure VI. Hydrogel swelling. (A) The syneresis results of hydrogels $10-21$. (B) Swelling data of hydrogel formulations over 25 days. After 12 days, the integrity of the hydrogels became compromised. Degree of swelling ranged from $0-150 \%$, with $\mathrm{CaCO}_{3}$ content significantly influencing the degree.

MB release from the hydrogel formulations is further detailed in Figure VI. Among all hydrogels, an initial burst release was observed within the first 5 days, with over 50\% MB released. Following the initial burst, a slower and more sustained release followed until the hydrogels disintegrated. Lower concentration hydrogels had the most cumulative MB release ( $\sim 90 \%)$ by 12 days, the point at which the alginate hydrogels were mostly dissolved and released remaining MB. 


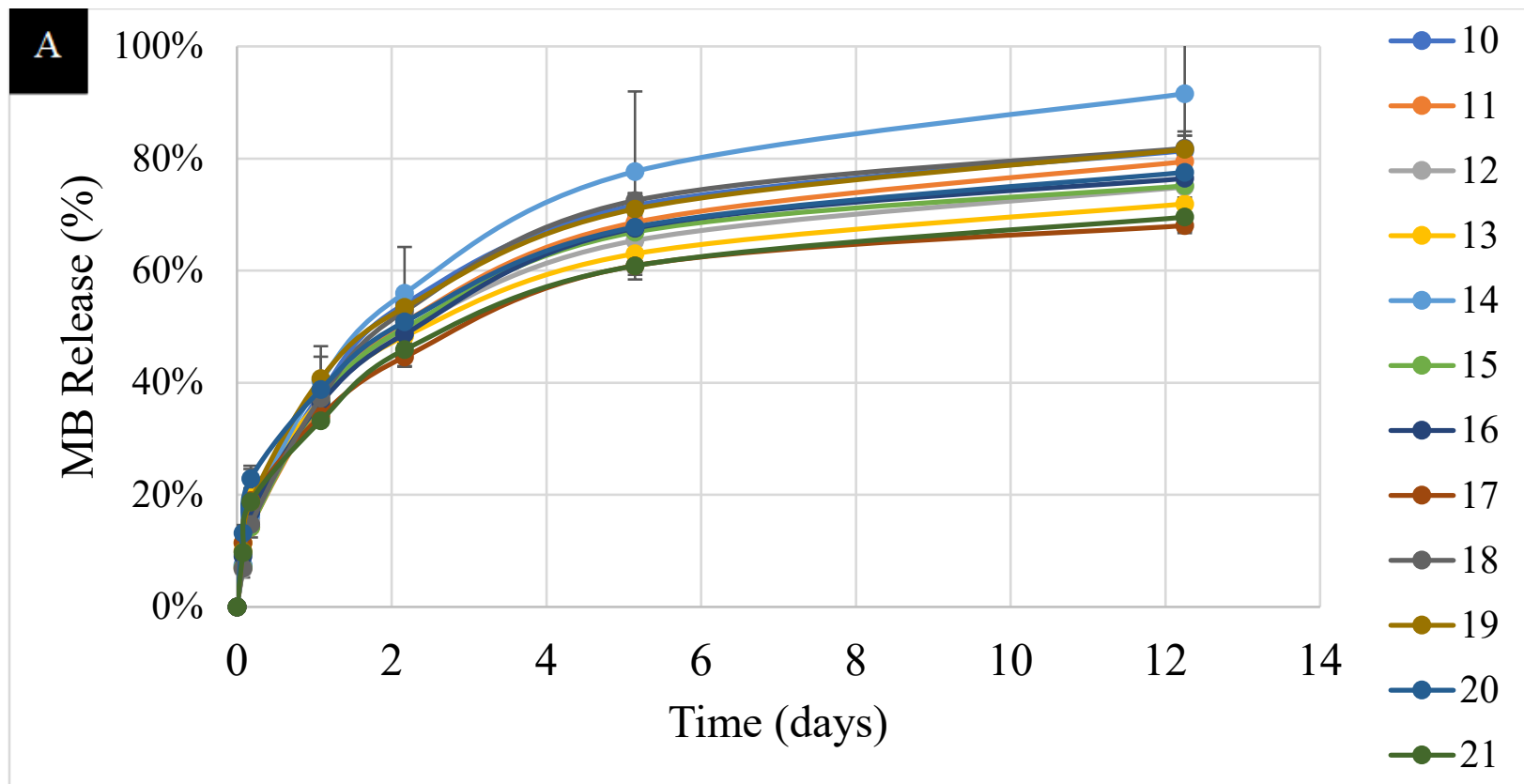

\section{B $100 \%$}

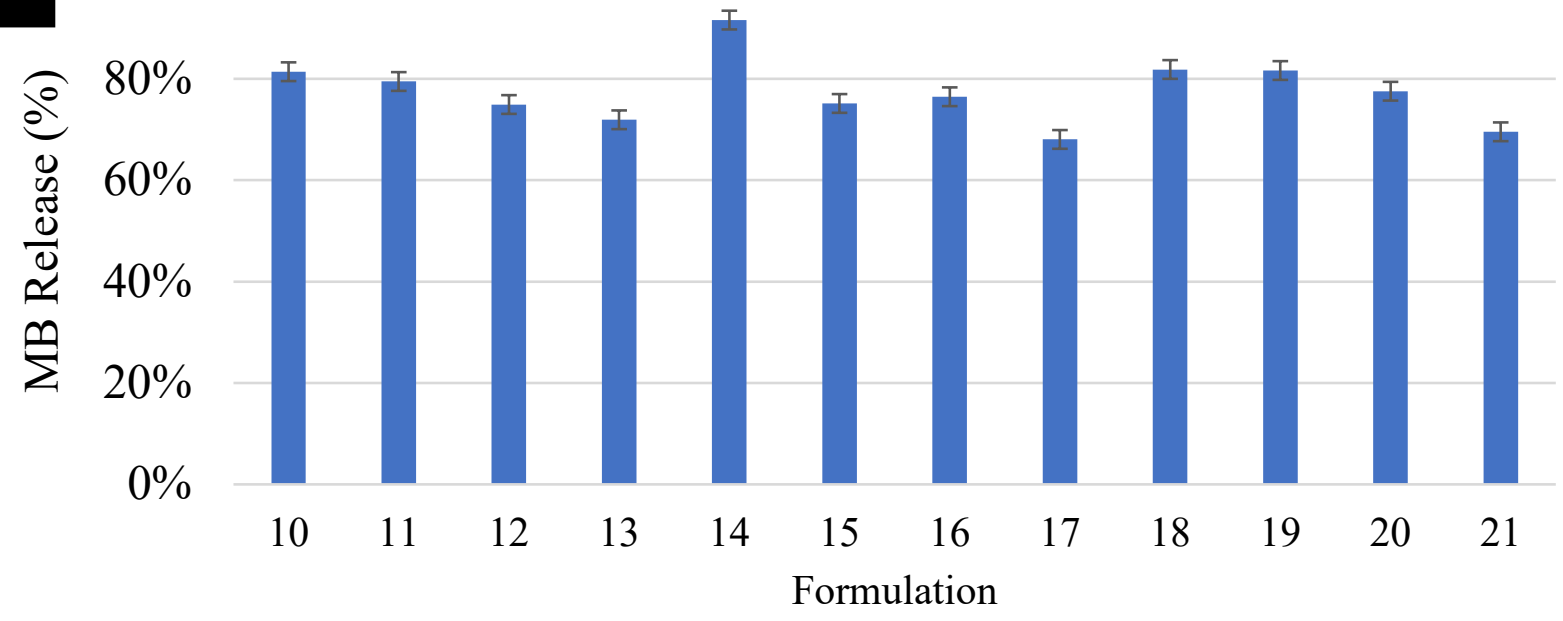

Figure VII. MB release. (A) The release profile of methylene blue of hydrogel formulations $10-21 .$. (B) The cumulative release of MB from hydrogels recorded at Day 12. Medium concentration hydrogels have the highest release profile of low and high concentration hydrogels at each time point

\subsection{Cytotoxicity of Alginate Hydrogels}

A fundamental requirement for injection is minimal cytotoxicity. To this end, following synthesis of representative hydrogels, we studied their biocompatibility using a human retinal cell line. ARPE-19 cells were incubated with the representative hydrogels for 24 hours and assessed via MTS assay. Formulations 10 and 21, demonstrated excellent cellular viability at over 95\%, whereas formulation 16 , had viability of $70 \%$ of following one-day exposure with the alginate hydrogels. Alginate hydrogels have proven to be biocompatible in various studies $[15,16]$ and our 
results using the different crosslinker further support biocompatibility as well as their potential for injection.

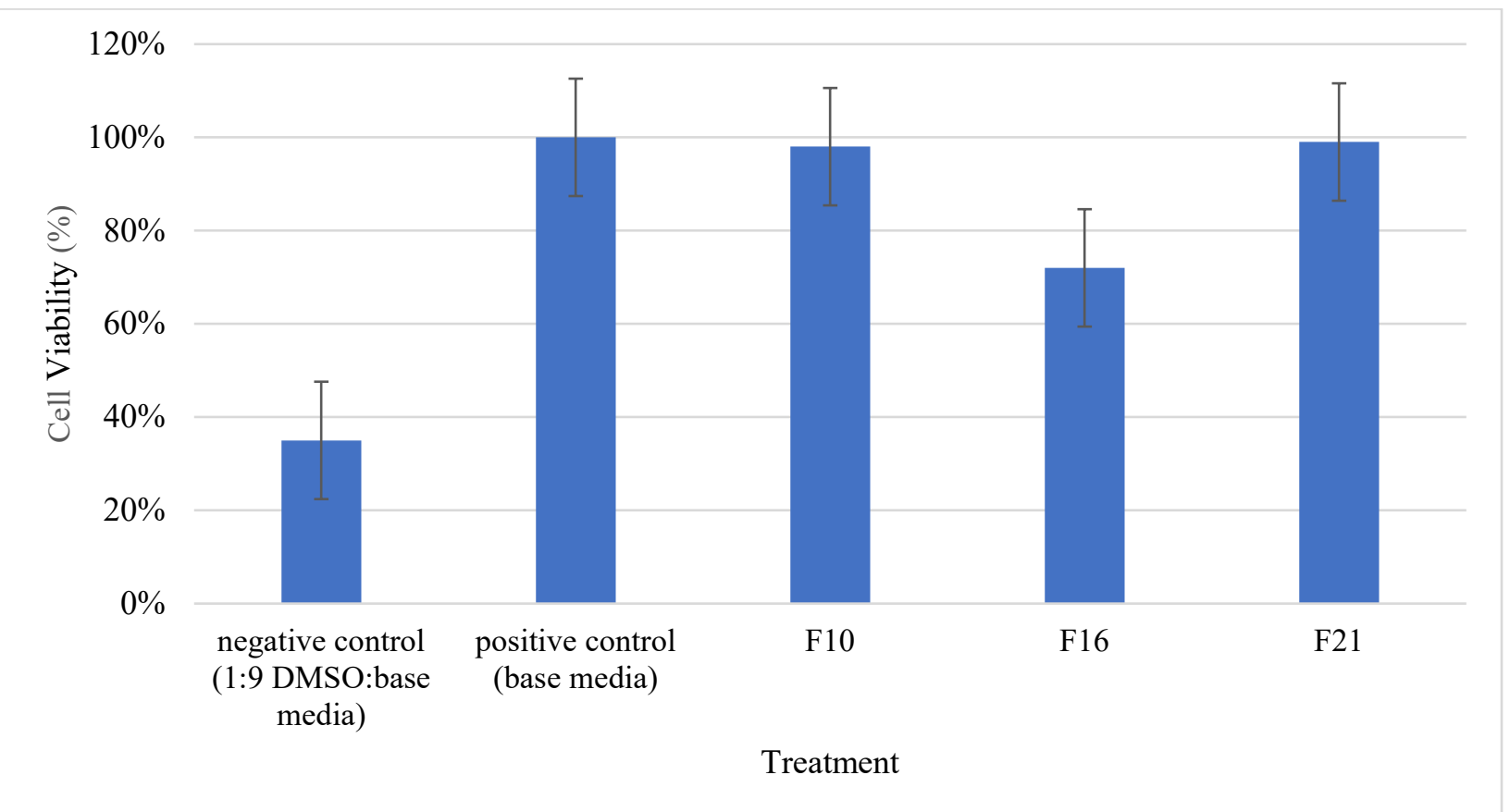

Figure VIII. Cytotoxicity of Representative Hydrogels. Cellular viability as measured by optical density (OD) of the MTS reagent product following exposure to alginate hydrogels. The low, medium, and high concentration hydrogels that were evaluated maintained a cell viability of at least $70 \%$ that of the positive control base media (DMEM/F12, 10\% FBS, 1\% PS).

\section{6. $M B$ as a ROS Scavenger}

Scavenging of ROS by MB was evaluated through in vitro testing based on published methods [27]. ARPE-19 cells were first incubated with MB concentrations of 0, 0.05, 0.25, 0.50, 1.0 and $2.0 \mathrm{mg} / \mathrm{mL}$ for 24 hours and then treated with $\mathrm{H}_{2} \mathrm{O}_{2}$ for 24 hours. ROS levels/activity was characterized by the appearance of highly fluorescent compound DCF in the DCFH-DA assay. There was an observable decrease in fluorescence of the cells corresponding to increased MB concentrations. We confirmed that ROS levels decreased significantly with concentrations of $0.500,1.00$ and $2.00 \mathrm{~g} / \mathrm{L}(\mathrm{p}<0.05)$ (Figure VIII). These results suggest the potential of using MB as ROS scavengers for TON treatment. 


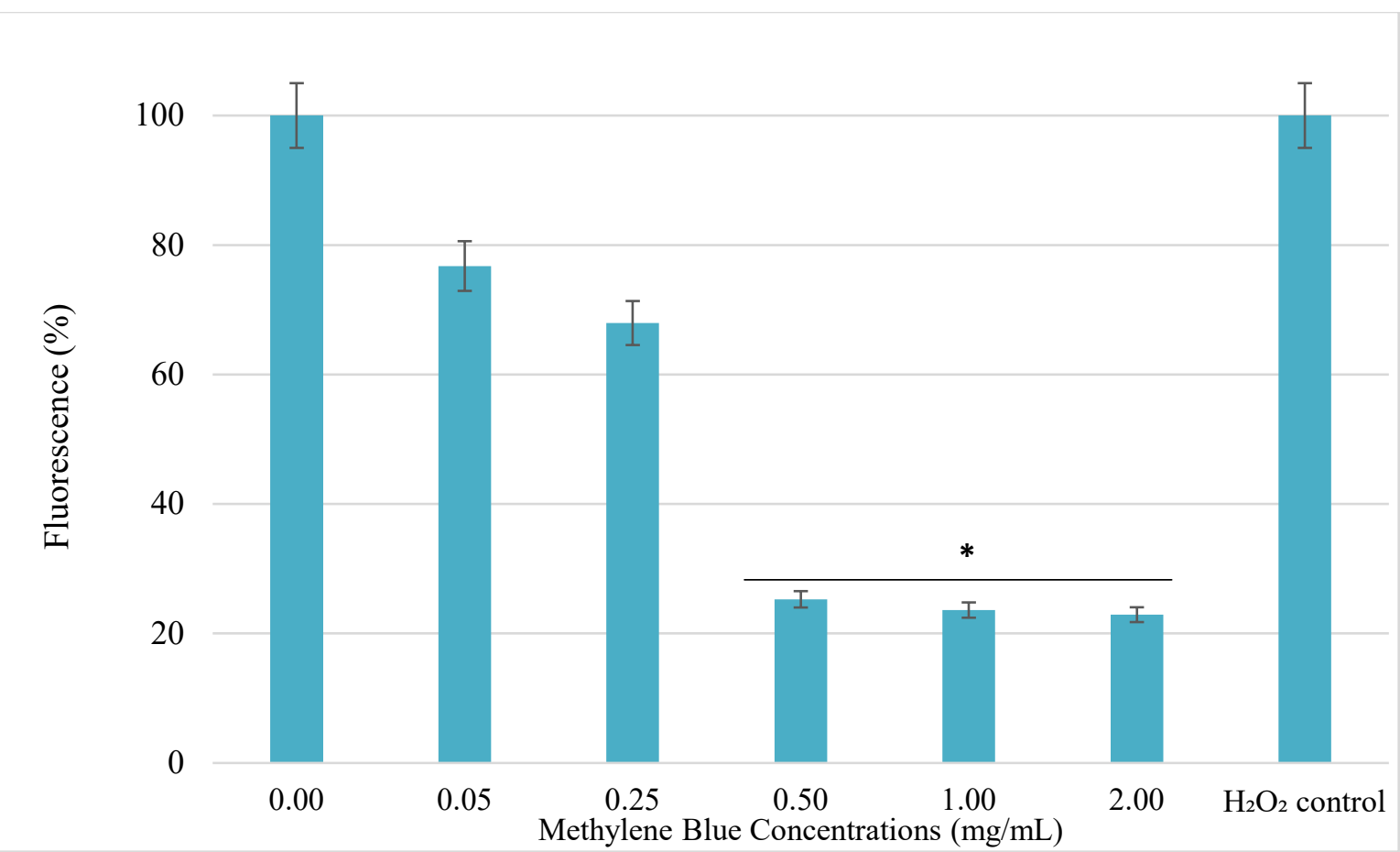

Figure IX. Methylene blue ROS results. ROS activity measured by DCF fluorescence in ARPE-19 cells induced by $600 \mu \mathrm{M} \mathrm{H}_{2} \mathrm{O}_{2}$. Increased concentrations of methylene blue contributed to higher cell survival during prolonged exposure to $\mathrm{H}_{2} \mathrm{O}_{2}$. Data $(n=5)$ is presented as mean \pm standard deviation. Results were normalized against $\mathrm{H}_{2} \mathrm{O}_{2}$ control. Higher fluorescence is indicative of greater DCF presence, more ROS activity and lowered cell survivability. Differences in the fluorescence of MB concentrations of $0.5,1.0$ and 2.0 were found to be statistically significant $\left({ }^{*} \mathrm{p}\right.$ $<0.05)$.

The ability to scavenge ROS was confirmed with MB. Additional studies were performed with alginate to further confirm MB's ROS scavenging ability while loaded into a hydrogel. All hydrogels were loaded with $1.0 \mathrm{~g} / \mathrm{L} \mathrm{MB}$, except the negative control 16, which as loaded without MB. ARPE-19 cells were incubated with hydrogel formulations 10, 16, 16 without MB and 21 for 24 hours. Following incubation, the hydrogels and cells were exposed to $\mathrm{H}_{2} \mathrm{O}_{2}$ for 24 hours with resulting DCF fluorescence measured. Hydrogels 10 and 21 displayed higher degrees of cell survival compared to 16 , yielding similar results to our cytotoxicity study. Low and high concentration alginate hydrogels $(10,21)$ achieved ARPE-19 survival of over $60 \%$ when exposed to the highly cytotoxic $\mathrm{H}_{2} \mathrm{O}_{2}$. Medium concentration hydrogels (16) maintained cell survival of $\sim 35 \%$ (with $\mathrm{MB}$ ); however, survival was lowered to $\sim 10 \%$ when cells were exposed to the hydrogels without MB. The presence of MB was found to significantly influence cell survival when loaded into hydrogel formulation $16(\mathrm{p}<0.01)$ as survival increased from $\sim 10 \%$ without MB to $35 \%$ with MB. 


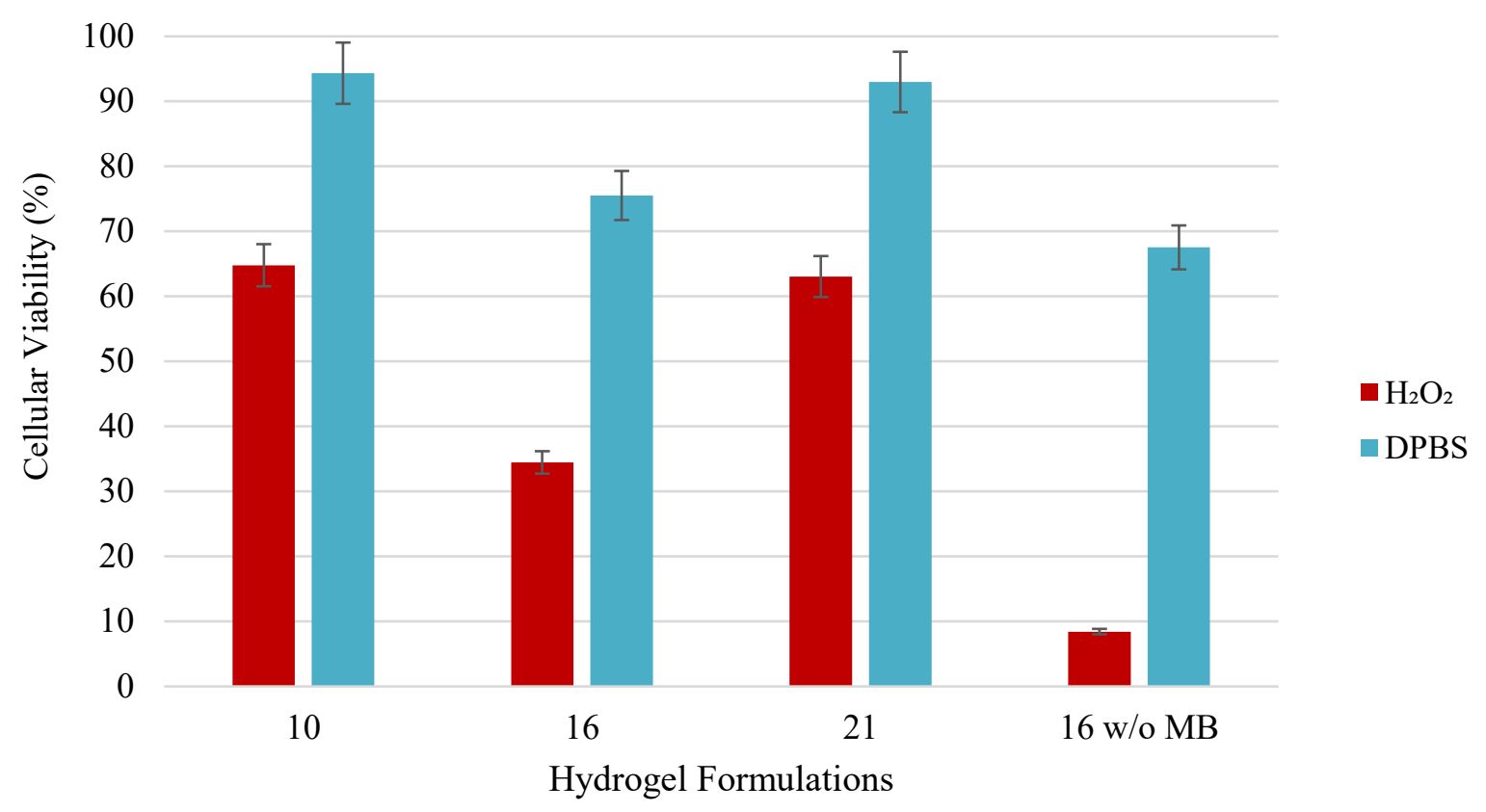

Figure X. Hydrogel ROS. Cell survival was maintained at over 50\% for formulations 10 and 21 . Cell survival did decrease following exposure to $\mathrm{H}_{2} \mathrm{O}_{2}$. Differences between 10 and 21 were found to not be statistically significant (p $>0.05)$. Differences between formulation 16 with and without MB was found to be statistically significant ( $p<0.01)$.

\subsection{Hydrogel Degradation}

Naturally derived biomaterials can be advantageous for drug delivery applications as their components can be broken down and removed by the body. Biodegradation of alginate can be more challenging than other biomaterials as it degrades by ion exchange. The in vitro degradation of alginate hydrogels was studied for two weeks with the mass of the initial and final mass recorded. Table III summarizes the degradation results of hydrogel formulations $10-21$. 
III. Hydrogel Degradation Results. The masses of hydrogel formulations $10-21$ were recorded over 14 days and weighed at $0,1,3,7$, and 14-day timepoints following lyophilization.

\begin{tabular}{cccccc}
\hline Formulation & Day 0 (mg) & Day $\mathbf{1}(\mathbf{m g})$ & Day 3 (mg) & Day $\mathbf{7}(\mathbf{m g})$ & Day 14 (mg) \\
\hline $\mathbf{1 0}$ & $6.4 \pm 0.5$ & $12.2 \pm 0.2$ & $11.5 \pm 0.8$ & $10.3 \pm 1.4$ & $24.9 \pm 2.2$ \\
$\mathbf{1 1}$ & $6.4 \pm 0.5$ & $13.5 \pm 1.0$ & $12.2 \pm 0.3$ & $10.5 \pm 0.7$ & $24.9 \pm 11.0$ \\
$\mathbf{1 2}$ & $8.0 \pm 0.5$ & $12.8 \pm 0.5$ & $11.7 \pm 0.3$ & $10.6 \pm 0.9$ & $13.7 \pm 1.4$ \\
$\mathbf{1 3}$ & $9.6 \pm 0.2$ & $14 . \pm 2.9$ & $12.2 \pm 0.8$ & $12.0 \pm 0.8$ & $21.3 \pm 6.0$ \\
$\mathbf{1 4}$ & $6.7 \pm 0.6$ & $13.8 \pm 1.8$ & $13.2 \pm 0.3$ & $11.1 \pm 0.4$ & $27.7 \pm 10.7$ \\
$\mathbf{1 5}$ & $7.7 \pm 0.2$ & $12.6 \pm 2.3$ & $11.4 \pm 1.1$ & $10.5 \pm 0.4$ & $11.8 \pm 0.6$ \\
$\mathbf{1 6}$ & $8.5 \pm 0.2$ & $12.3 \pm 1.3$ & $11.0 \pm 0.1$ & $11.4 \pm 0.7$ & $13.8 \pm 2.7$ \\
$\mathbf{1 7}$ & $9.9 \pm 0.3$ & $11.4 \pm 0.6$ & $11.8 \pm 3.2$ & $11.4 \pm 0.7$ & $16.1 \pm 1.9$ \\
$\mathbf{1 8}$ & $7.2 \pm 0.5$ & $13.7 \pm 0.5$ & $12.3 \pm 1.1$ & $12.9 \pm 7.5$ & $14.7 \pm 5.4$ \\
$\mathbf{1 9}$ & $7.8 \pm 0.3$ & $12.8 \pm 0.5$ & $11.1 \pm 0.9$ & $17.6 \pm 3.0$ & $14.0 \pm 1.3$ \\
$\mathbf{2 0}$ & $8.5 \pm 0.5$ & $12.9 \pm 2$. & $12.4 \pm 2.6$ & $13.4 \pm 1.1$ & $15.5 \pm 3.6$
\end{tabular}

Swelling rates varied significantly on Days 0 and 14 based on hydrogel composition. Higher concentration hydrogels displayed the highest degree of swelling on Day 0; however, on Day 14, low concentration hydrogels displayed the highest degree of swelling. The average mass of the hydrogels $(\mathrm{mg})$ following $0,1,3,7$, and 14 days were $8.0 \pm 1.2,12.7 \pm 1.0,12.0 \pm 0.76,12.3 \pm$ 2.4 , and $18.3 \pm 5.4$, respectively. On days 1,3 and 7 of incubation, the degree of swelling as well as the average mass did not differ significantly from previous time points. Low concentration hydrogels among all time points displayed the lowest degree of swelling over time whereas higher concentration hydrogels had the highest degree of swelling, as expected. $\mathrm{CaCO}_{3}$ values were found to influence swelling over time with the lowest $\mathrm{GDL}: \mathrm{CaCO}_{3}$ ratio swelling most rapidly. The ratio of $\mathrm{Ca}^{2+}$ :alginate was the primary driver of hydrogel swelling with the highest ratios swelling the most overall. Hydrogels prepared with $\mathrm{Ca}^{2+}$ :alginate ratio of 0.50 were at approximately equilibrium swelling when formed.

The differences between the hydrogels among timepoints were not significant until Day 14 ( $\mathrm{p}<$ 0.05), with observable groupings between the low, medium and high concentration hydrogels. Low and high concentration hydrogels displayed the highest degrees of swelling in this timepoint (Day 14). Day 0 differed significantly among all timepoints $(\mathrm{p}<0.001)$; Day 1 differed 
significantly from Day $14(\mathrm{p}=0.0020)$ and Day 7 differed significantly from Day $14(\mathrm{p}=$ $0.0021)$.

\section{Discussion and Conclusion}

In this study, to address the shortage of treatment options for TON, we developed an injectabledrug loaded delivery vehicle to release MB around the optic nerve following injury. Sustainable MB release was achieved among all synthesized alginate hydrogel formulations and may be able to be used for optic neuropathy treatments.

As reported in Table I, alginate hydrogels were synthesized by internal crosslinking with evenly distributed insoluble calcium carbonate and the slow hydrolyzing proton donor, GDL. This method slows down the rate of gelation to $\sim 10-50 \mathrm{~min}$, accommodating adequate mixing of all components and subsequent injection through a small gauge needle.

Hydrogel formulations $1-9$ were prepared based on their ability to consistently form solid hydrogels. Molar ratios of GDL: $\mathrm{CaCO}_{3}$ were found to influence $\mathrm{pH}$ values of the hydrogels, with concentrations greater than one contributing to a decrease in $\mathrm{pH}$ values. Formulations $10-21$ were created based on $1-9$, as well as demonstrating physiological $\mathrm{pH}$ values of $6-8$.

Calcium ions form ionic crosslinks with alginate polymers by attracting the carboxyl group from two adjacent alginate monomers between two polymer chains. Hypothetically, maximum crosslinking would occur when four moles of alginate monomer are present for every one mole of $\mathrm{Ca}^{2+}$. All $\mathrm{CaCO}_{3}$ concentrations of hydrogel formulations in this study were prepared based on $\mathrm{Ca}^{2+}$ :alginate molar ratios slightly less than, equal to and greater than this crosslinking maximum. Hydrogel formulations were evaluated by rheological testing. Results confirmed the gel-like nature of the alginate hydrogels (G'> G') and all reached gelation within an hour. Viscoelasticity was influenced by the ratios of GDL: $\mathrm{CaCO}_{3}$ and the weight percent of alginate. Hydrogels with low concentrations of GDL and $\mathrm{CaCO}_{3}$ had G' values of around $20 \mathrm{~Pa}$, medium gels $\sim 35 \mathrm{~Pa}$ and high concentration gels of $\sim 95 \mathrm{~Pa}(\mathrm{p}<0.05)$. Hydrogels were targeted to mimic the mechanical properties of soft nerve tissues. As expected, a higher $\mathrm{Ca}^{2+}$ concentration induces stronger gelation [29] which is evident in our results. Future studies may focus on increasing $\mathrm{Ca}^{2+}$ concentrations to higher concentration levels to increase modulus. Additionally, higher concentration hydrogels 
gelled significantly faster than low and medium hydrogels $(\mathrm{p}<0.05)$, with formulations 10 and 21 having the slowest and most rapid gelation time, respectively.

ARPE-19 cells were used to represent the microenvironment of the optic nerve because although primary ON cells would be preferred, their usage is limited due to difficulty in obtaining/isolating and slow proliferation rates [30]. As such, the immortalized cell line ARPE-19 was chosen in our study. Cytotoxicity results indicated that the hydrogels demonstrated low to minimal toxicity with low and high GDL: $\mathrm{CaCO}_{3}$ concentration hydrogels preferred over medium hydrogels. Medium concentration hydrogels were found to be more cytotoxic than low and high concentration hydrogels $(70 \%$ survival compared to $>90 \%)$ potentially due to the $\mathrm{Ca}^{2+:}$ alginate molar ratio crosslinking maximum. Results are consistent with previous reports of the use of alginate hydrogels and MB for drug delivery and tissue engineering applications $[8-12,15,16]$.

The scavenging ability of $\mathrm{MB}$ was validated through various concentrations of $\mathrm{MB}$ and low, medium and high concentration hydrogels and evaluated by DCF assay. ROS results indicate that MB concentrations of 0.50, 1.00, and 2.00 reduced ROS activity. Additionally, low and high concentration hydrogels demonstrated higher survival of ARPE-19 cells. Results were consistent with MB's ability to scavenge ROS.

In conclusion, to improve upon the current management options for TON, internally crosslinked sodium alginate hydrogels were developed. We also identified the hydrogel with optimal mechanical properties and drug release by modulating its components using design of experiments. The designed alginate drug delivery system is biocompatible and adequately lowers the concentration of reactive oxygen species in vitro. Most importantly, the proposed design improves upon the current treatments for TON. Given the results of the drug release as well as its biocompatibility and injectability, high concentration alginate hydrogels have the potential to improve TON damage as well as other diseases in which there is an accumulation of reactive species, which will be validated in vivo in future studies.

\section{Ethical Statements}

o Ethics approval and consent to participate: Not Applicable

o Consent for publication: All authors agreed with the content and give their consent to submit the manuscript for publication. 
o Availability of data and materials: The data generated during and/or analyzed during this study are available on request from the corresponding author.

o Competing interests: The authors declare that they have no competing interests related to this work.

o Funding: Supported by the US Department of Defense Vision Research Program Award W81XWH-15-1-0074. The opinions or assertions contained herein are the private views of the authors and are not to be construed as official or as reflecting the views of the Department of the Army or the Department of Defense.

o Authors' contributions: C.M., A.S., M.R., and K.S.-R. planned the experiments. C.M., A.S., W.R., and A.C. carried out the experiments. C.M., A.S., M.R., and K.S.-R. contributed to the interpretation of the results. C.M., A.S., M.R., and K.S.-R. drafted the manuscript. M.R. and K.S.-R. conceived the study and were in charge of overall direction and planning.

o Acknowledgements: The authors would like to acknowledge undergraduate students Emma McLaughlin, Marissa Ruzga, and Samantha Thobe, who contributed to preliminary studies and data visualization. We would also like to thank Dr. Aleksander Skardal for providing fluorescent plate readers.

o Authors' information: Corresponding Author: Katelyn E. Swindle-Reilly, Department of Biomedical Engineering, The Ohio State University, 3010 Fontana Labs, $140 \mathrm{~W} 19$ th Ave, Columbus, OH 43210,614-292-4602, reilly.198@,osu.edu

\section{References}

1. Steinsapir, K. D. \& Goldberg, R. A. Traumatic Optic Neuropathy. Survey of Ophthalmology 38, 487-518 (1994).

2. Pirouzmand, F. Epidemiological Trends of Traumatic Optic Nerve Injuries in the Largest Canadian Adult Trauma Center. Journal of Craniofacial Surgery 23, 516-520 (2012).

3. Weichel, E. D., Colyer, M. H., Bautista, C., Bower, K. S. \& French, L. M. Traumatic Brain Injury Associated with Combat Ocular Trauma. Journal of Head Trauma Rehabilitation 24, 41-50 (2009).

4. Levin, L. a, Beck, R. W., Joseph, M. P., Seiff, S. \& Kraker, R. The Treatment of Traumatic Optic Neuropathy: The International Optic Nerve Trauma Study. Ophthalmology 106, 1268-77 (1999).

5. Wells, J. et al. Early in vivo changes in calcium ions, oxidative stress markers, and ion channel immunoreactivity following partial injury to the optic nerve. Journal of Neuroscience Research $\mathbf{9 0}$, 606-618 (2012).

6. O'Hare Doig, R. L. et al. Reactive species and oxidative stress in optic nerve vulnerable to secondary degeneration. Experimental Neurology 261, 136-146 (2014).

7. Xiong, Z. M. et al. Methylene blue alleviates nuclear and mitochondrial abnormalities in progeria. Aging Cell 15, 279-290 (2016). 
8. Ryou, M. G. et al. Methylene blue-induced neuronal protective mechanism against hypoxiareoxygenation stress. Neuroscience 301, 193-203 (2015).

9. Duan, Y., Haugabook, S. J., Sahley, C. L. \& Muller, K. J. Methylene Blue Blocks cGMP Production and Disrupts Directed Migration of Microglia to Nerve Lesions in the Leech CNS. Journal of Neurobiology 57, 183-192 (2003).

10. Wiklund, L. et al. Neuro- And Cardioprotective Effects of Blockade of Nitric Oxide Action by Administration of Methylene Blue. Annals of the New York Academy of Sciences 1122, 231-244 (2007).

11. $\mathrm{Xu}, \mathrm{H}$. et al. Methylene blue attenuates neuroinflammation after subarachnoid hemorrhage in rats through the Akt/GSK-3ß/MEF2D signaling pathway. Brain, Behavior, and Immunity 65, 125-139 (2017).

12. Shen, Q. et al. Neuroprotective efficacy of methylene blue in ischemic stroke: An MRI study. PLoS One 8, 1-6 (2013).

13. Talley Watts, L. et al. Methylene Blue Is Neuroprotective against Mild Traumatic Brain Injury. Journal of Neurotrauma 31, 1063-1071 (2014).

14. Tønnesen, H. H. \& Karlsen, J. Alginate in Drug Delivery Systems. Drug Development and Industrial Pharmacy 28, 621-630 (2002).

15. Sun, J. \& Tan, H. Alginate-based Biomaterials for Regenerative Medicine Applications. Materials (Basel) 6, 1285-1309 (2013).

16. Draget, K. I., Ostgaard, K. \& Smidsrod, O. Alginate-based solid media for plant tissue culture. Applied Microbiology and Biotechnology 31, 79-83 (1989). Kuo, C. K. \& Ma, P. X. Maintaining dimensions and mechanical properties of ionically crosslinked alginate hydrogel scaffolds in vitro. Journal of Biomedical Materials Research Part A 84, 899-907 (2008).

17. 21 Braccini, I. \& Pérez, S. Molecular Basis of $\mathrm{Ca}^{2+}$-Induced Gelation in Alginates and Pectins: The Egg-Box Model Revisited. Biomacromolecules 2, 1089-1096 (2001).

18. Kuo, C. K. \& Ma, P. X. Maintaining dimensions and mechanical properties of ionically crosslinked alginate hydrogel scaffolds in vitro. Journal of Biomedical Materials Research Part A 84, 899-907 (2008).

19. Kuo, C. K. \& Ma, P. X. Ionically Crosslinked Alginate Hydrogels as Scaffolds for Tissue Engineering: Part 1. Structure, Gelation Rate and Mechanical Properties. Biomaterials 22, 511$521(2001)$.

20. Draget, K. I., Østgaard, K. \& Smidsrød, O. Homogeneous Alginate Gels: A Technical Approach. Carbohydrate Polymers 14, 159-178 (1990).

21. 28 Gomathi, T., Susi, S., Abirami, D. \& Sudha, P. N. Size Optimization and Thermal Studies on Calcium Alginate Nanoparticles. (2017).

22. Zuidema, J.M., Rivet, C.J., Gilbert, R.J. \& Morrison, F.A. A protocol for rheological characterization of hydrogels for tissue engineering strategies. J. Biomed. Mater. Res. - Part B Appl. Biomater. 102, 1063 - 10773 (2014).

23. Park, H. et al. Effect of Swelling Ratio of Injectable Hydrogel Compositions on Chondrogenic Differentiation of Encapsulated Rabbit Marrow Mesenchymal Stem Cells In Vitro. Biomacromolecules 10, 541 - 546 (2009).

24. Niu, H. et al. Thermosensitive, fast gelling, photoluminescent, highly flexible, and degradable hydrogels for stem cell delivery. Acta Biomaterialia 83, 96-108 (2019).

25. Stoppel, W. L. et al. Terminal sterilization of alginate hydrogels: Efficacy and impact on mechanical properties. Journal of Biomedical Materials Research Part B: Applied Biomaterials 102, 877-884 (2013). doi:10.1002/jbm.b.33070

26. Violante, G. Da et al. Evaluation of the Cytotoxicity Effect of Dimethyl Sulfoxide (DMSO) on Caco2 / TC7 Colon Tumor Cell Cultures. Biological and Pharmaceutical Bulletin 25, 1600-1603 (2002).

27. Jiang, P., Choi, A., Swindle-Reilly, K.E. Controlled release of anti-VEGF by redox-responsive polydopamine nanoparticles. Nanoscale 12, 17298-17311 (2020). 
28. Voloboueva, L. A., Liu, J., Suh, J. H., Ames, B. N. \& Miller, S. S. (R)- $\alpha$-Lipoic Acid Protects Retinal Pigment Epithelial Cells from Oxidative Damage. Investigative Ophthalmology and Visual Science 46, 4302-4310 (2005).

29. Cuomo, F., Cofelice, M., Lopez, F. Rheological Characterization of Hydrogels from Alginate Based Nanodispersion. Polymers (Basel) 11, 259-270 (2019).

30. Liu, Y., Patel. G.C., Mao, W., Clark, A.F. Establishment of a conditionally immortalized mouse optic nerve astrocyte line. Experimental Eye Research 176, 188-195 (2018).

31. K. Y. Lee, D. J. M. Alginate: properties and biomedical applications. Progress in Polymer Science 37, 106-126 (2012). 
Figures

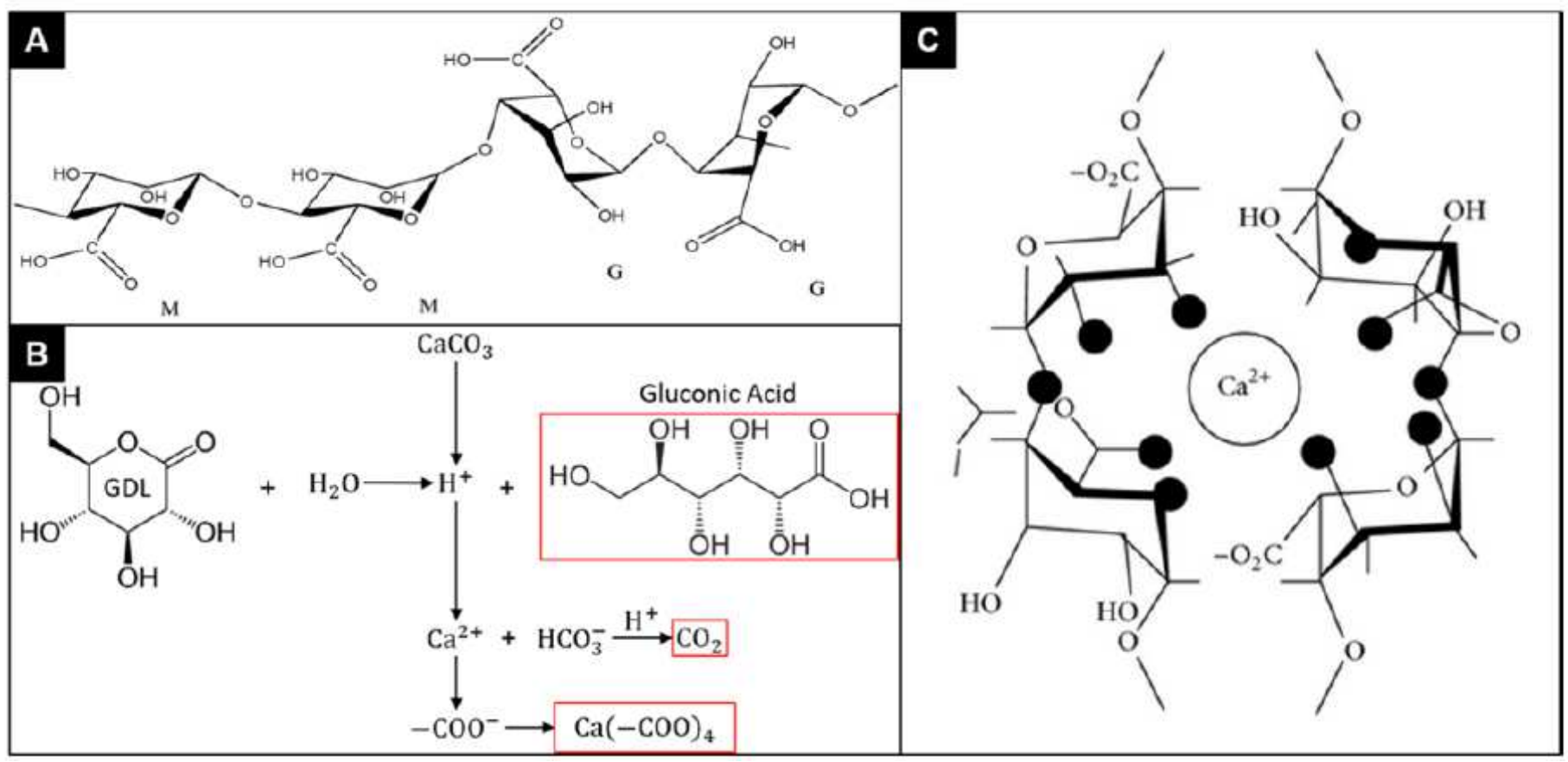

Figure 1

Diagram of crosslinking reaction and final hydrogel structure. [22] (A) Alginate is a polysaccharide copolymer composed of two residues, (1-4)-linked $\beta$-D mannuronate (M), and a-L-guluronate (G). The patterning and ratio of these residues can significantly impact the material properties of hydrogels. (B) Schematic of the crosslinking reaction between the proton donor D-glucono-lactone (GDL), the calcium ion source $\mathrm{CaCO} 3$ and the alginate polymer. The reaction generates three products - gluconic acid, carbon dioxide, and the calcium ion-alginate complex. (C) [18] Once $\mathrm{Ca} 2+$ is freed by GDL, the free ion interacts with alginate's carboxyl group to from ionic crosslinking between polymers. 

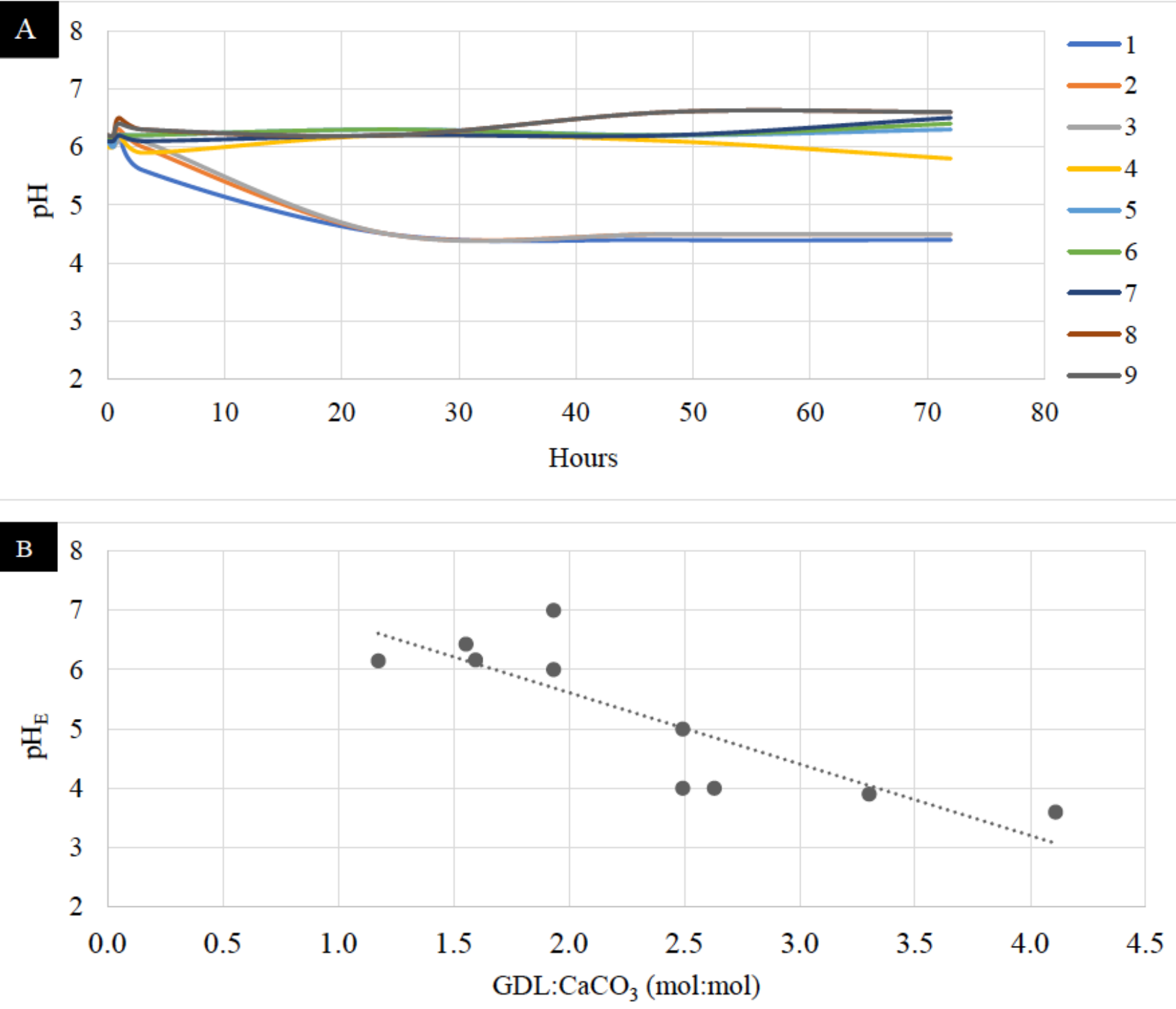

Figure 2

Characterization of $\mathrm{pH}$ of hydrogel formulations 1 - 9. (A) Evolution of hydrogel pH over 72 hours. Formulations exhibit clear groupings of $\mathrm{pH}$ values. (B) Plot of hydrogel equilibrium $\mathrm{pH}(\mathrm{pHE})$ reached after 72 hours of gelation. There is a linear and inverse relationship between GDL:CaCO3 and pHE with an R2 of $0.8(p<0.0001)$. 


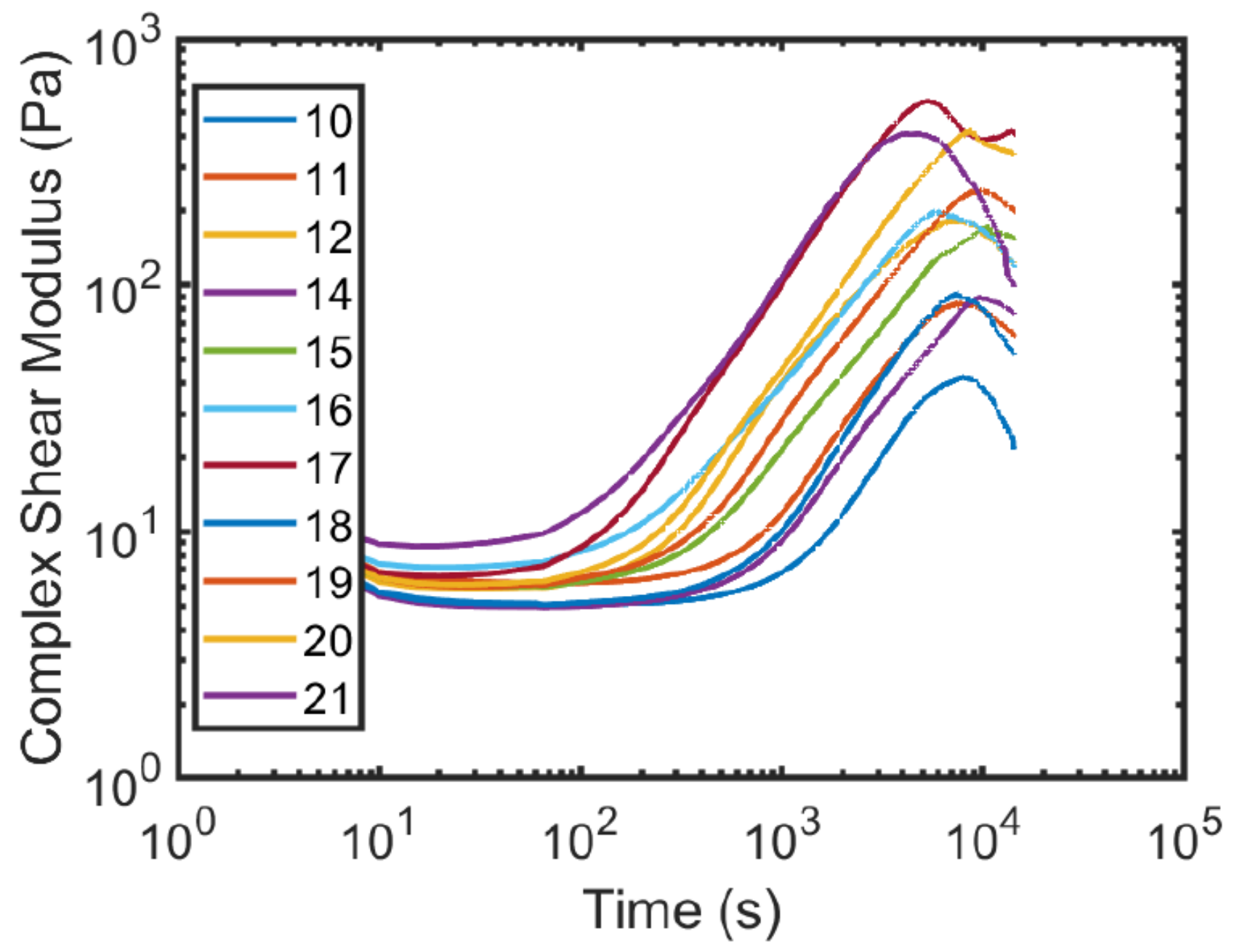

Figure 3

Gelation characterization of hydrogel formulations. Time sweep results of hydrogel formulations $10-21$, excluding 13. Formulations had observable groupings of low and high GDL:CaCO3 ratio hydrogels. Gelation times ranged from $707 \pm 59$ to $2803 \pm 40$ seconds. 

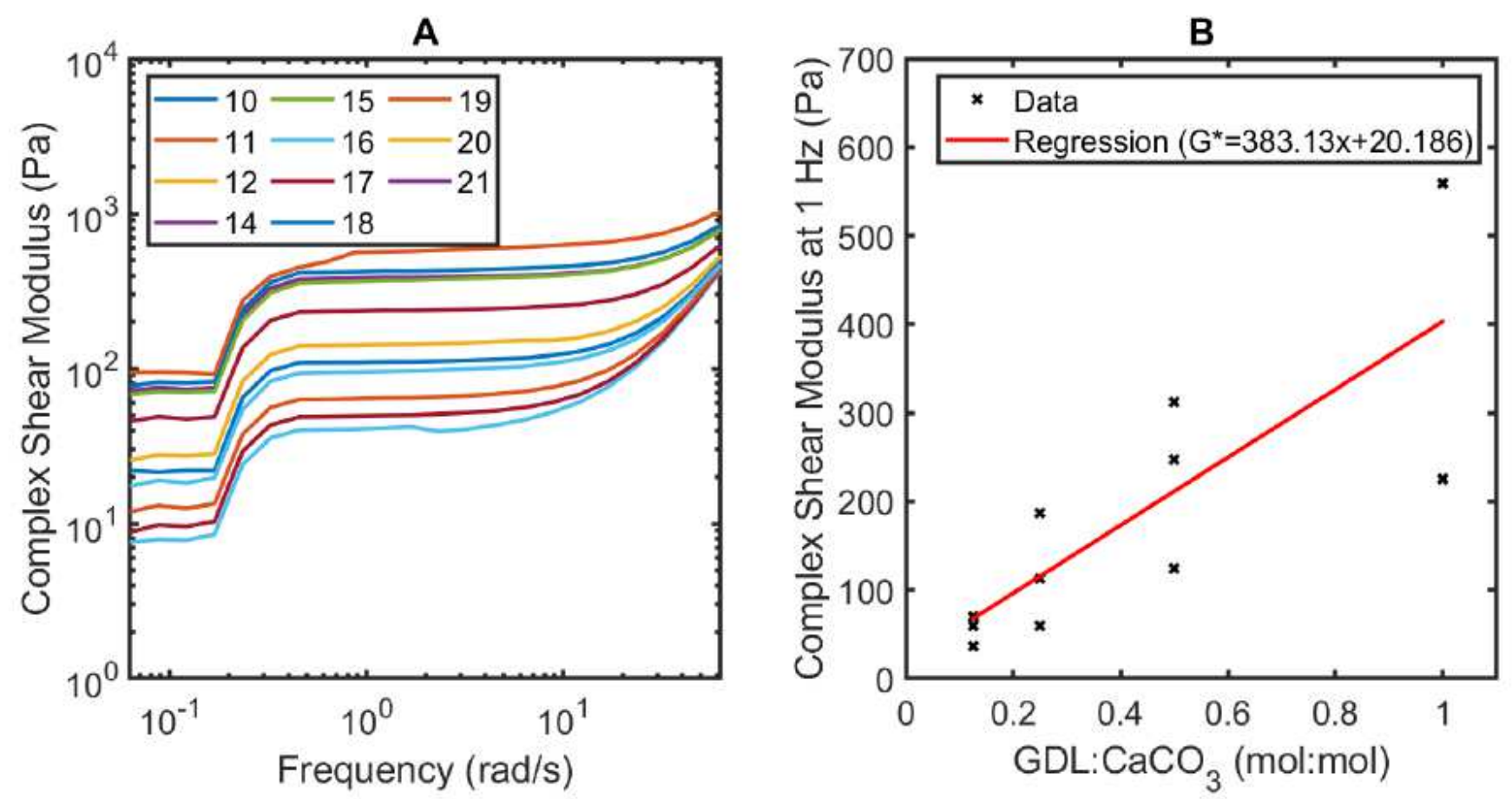

\section{Figure 4}

Frequency sweep results of hydrogel formulations. (A) Frequency sweep data from hydrogel formulations 10-21 (except 13). There is a positive exponential relationship between increasing frequency and complex shear stress. (B) Complex shear stress $\left(\mathrm{G}^{*}\right)$ as a function of concentration ratio (CaCO3:GDL) from hydrogel formulations $10-21$ at low frequencies. GDL:CaCO3 ratios significantly influence $\mathrm{G}^{\star}$, with higher ratios contributing to high complex shear stresses $(p<0.05)$.
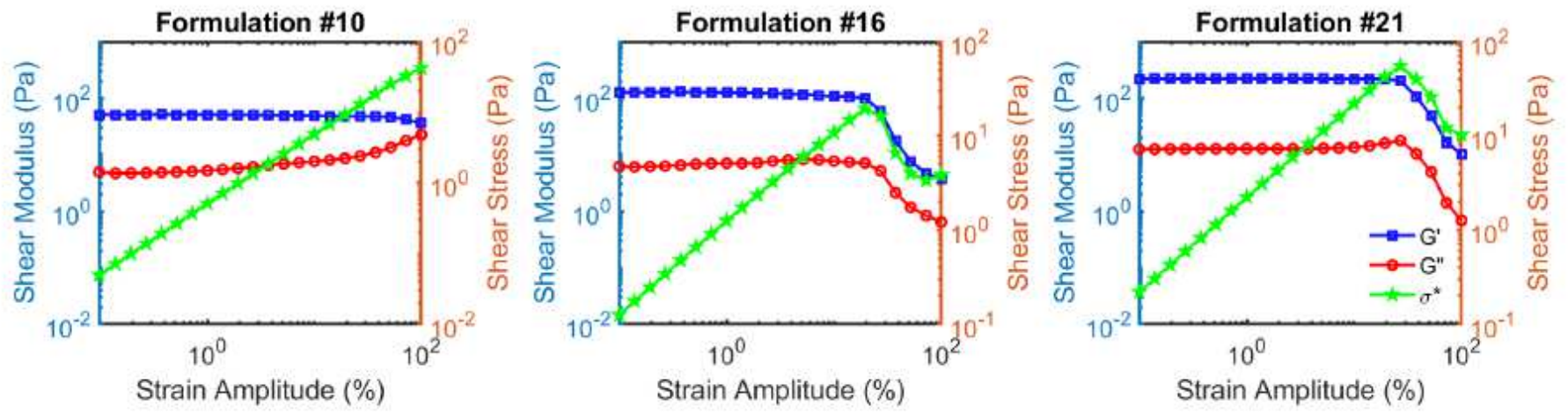

Figure 5

Rheological characterization of hydrogel formulations. Amplitude sweep data from representative hydrogels. A linear viscoelastic region of stiffness response corresponding to $1 \mathrm{~Hz}$ dynamic shear is observed up to $1 \%$ strain. 
A

1.2

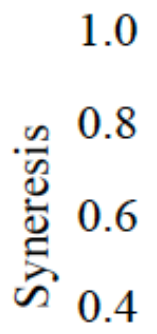

0.2

0.0
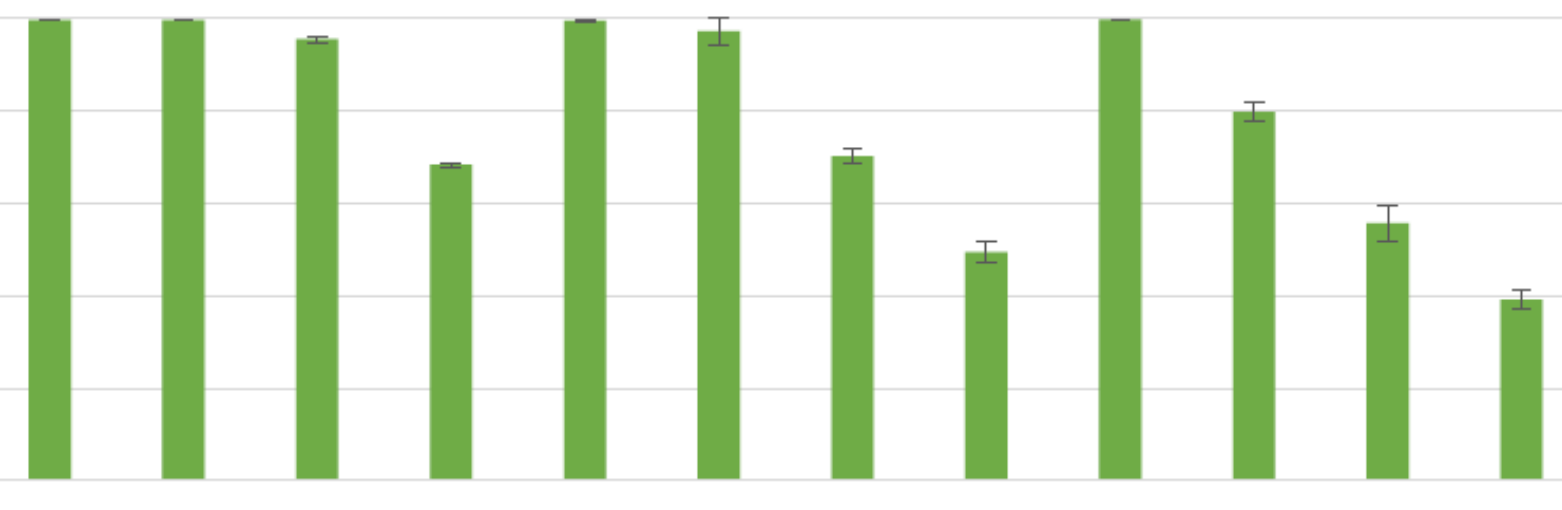

$\begin{array}{llllllllllll}10 & 11 & 12 & 13 & 14 & 15 & 16 & 17 & 18 & 19 & 20 & 21\end{array}$

Formulations

B

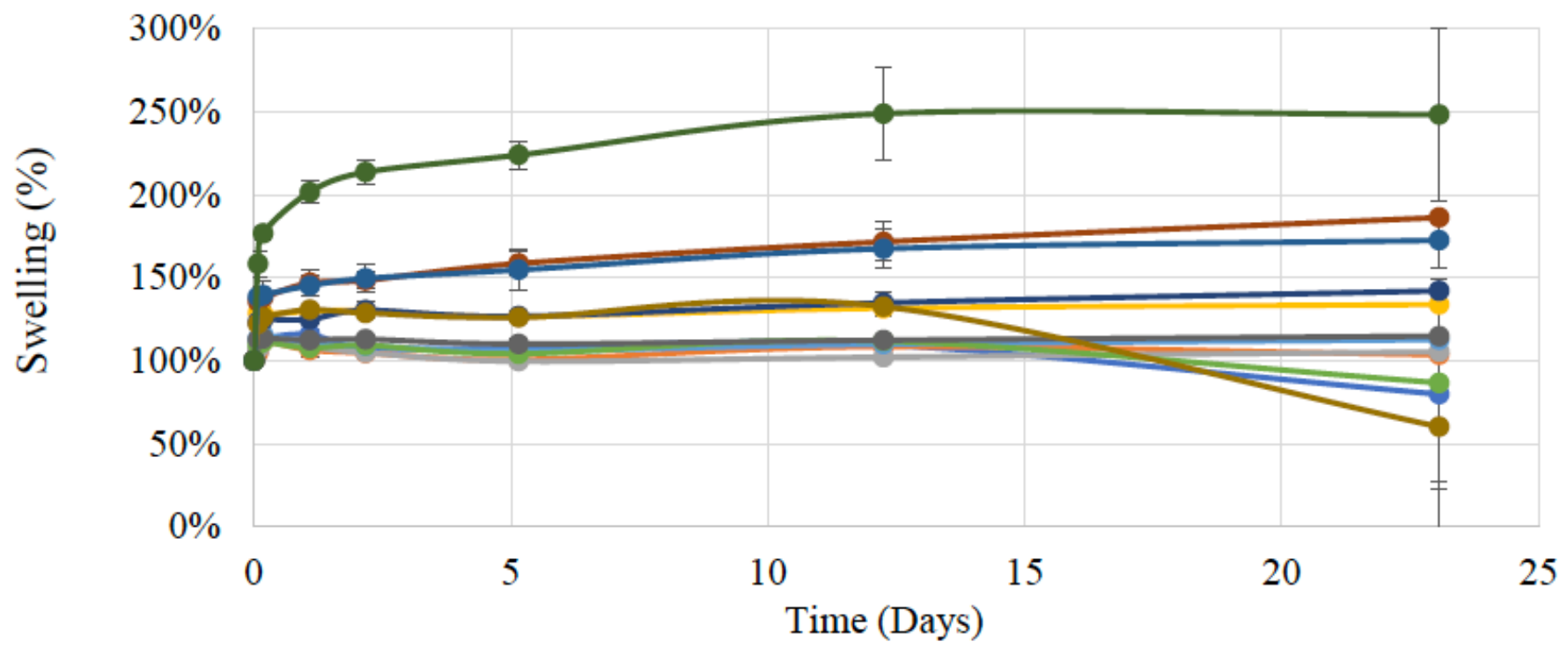

$-10$

$\multimap 11 \multimap 12 \multimap-13 \multimap 14$

$19-20$

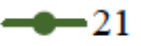

Figure 6

Hydrogel swelling. (A) The syneresis results of hydrogels $10-21$. (B) Swelling data of hydrogel formulations over 25 days. After 12 days, the integrity of the hydrogels became compromi 

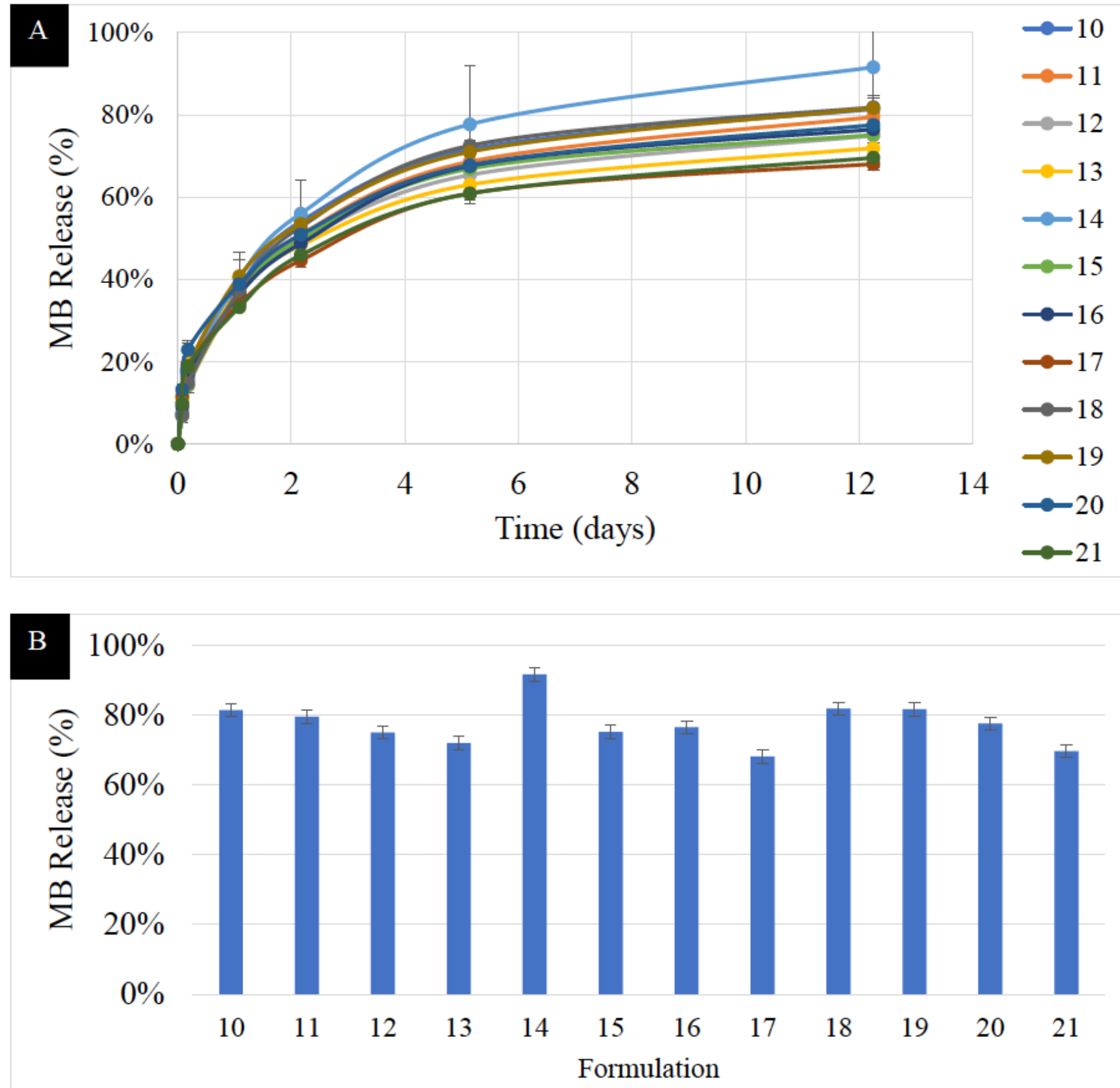

Figure 7

MB release. (A) The release profile of methylene blue of hydrogel formulations $10-21$.. (B) The cumulative release of MB from hydrogels recorded at Day 12. Medium concentration hydrogels have the highest release profile of low and high concentration hydrogels at each time point 


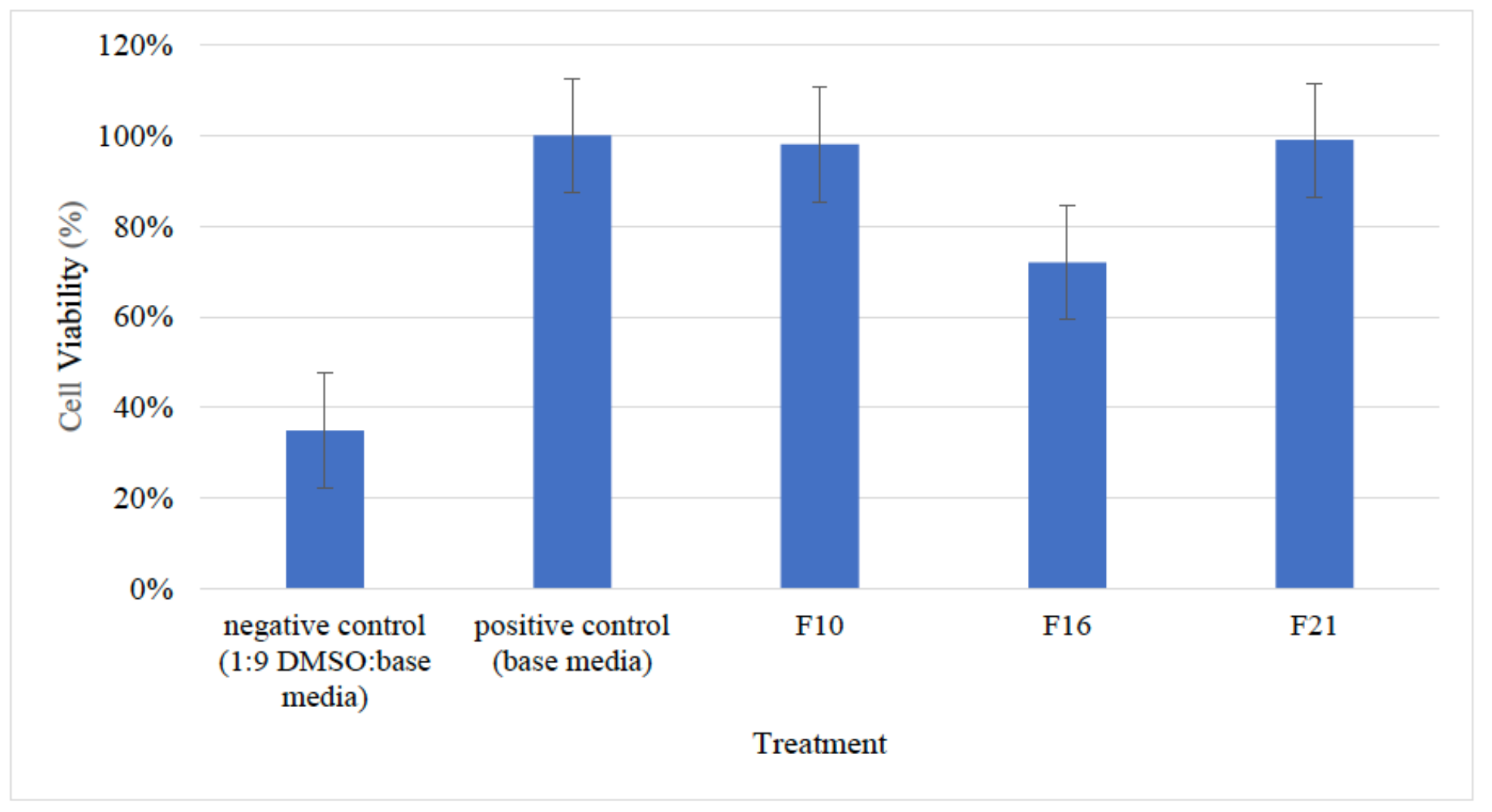

Figure 8

Cytotoxicity of Representative Hydrogels. Cellular viability as measured by optical density (OD) of the MTS reagent product following exposure to alginate hydrogels. The low, medium, and high concentration hydrogels that were evaluated maintained a cell viability of at least $70 \%$ that of the positive control base media (DMEM/F12, 10\% FBS, 1\% PS). 


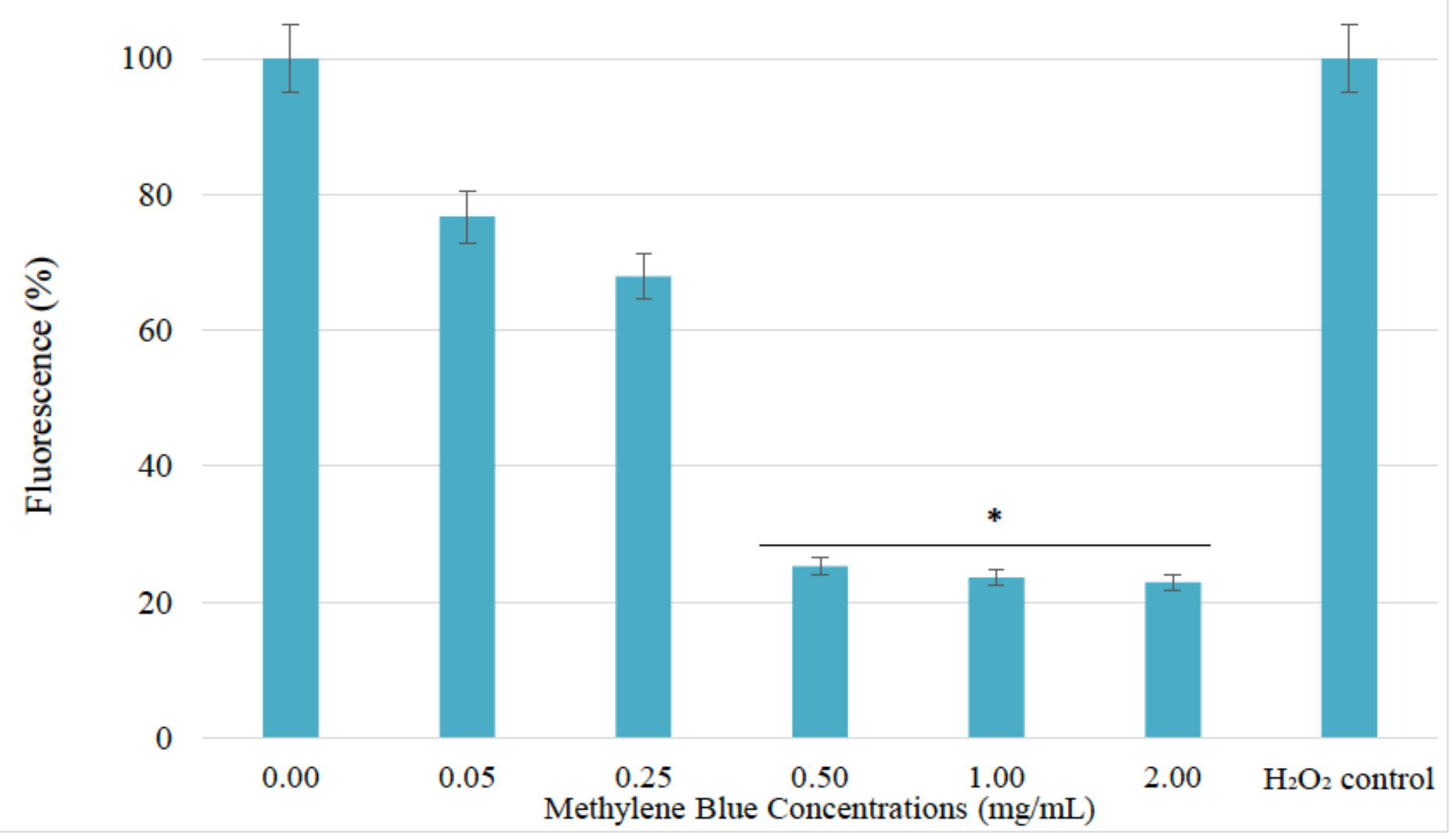

\section{Figure 9}

Methylene blue ROS results. ROS activity measured by DCF fluorescence in ARPE- 19 cells induced by 600 $\mu \mathrm{M} \mathrm{H} 2 \mathrm{O} 2$. Increased concentrations of methylene blue contributed to higher cell survival during prolonged exposure to $\mathrm{H} 2 \mathrm{O} 2$. Data $(n=5)$ is presented as mean \pm standard deviation. Results were normalized against $\mathrm{H} 2 \mathrm{O} 2$ control. Higher fluorescence is indicative of greater DCF presence, more ROS activity and lowered cell survivability. Differences in the fluorescence of MB concentrations of $0.5,1.0$ and 2.0 were found to be statistically significant $\left({ }^{*} p<0.05\right)$. 


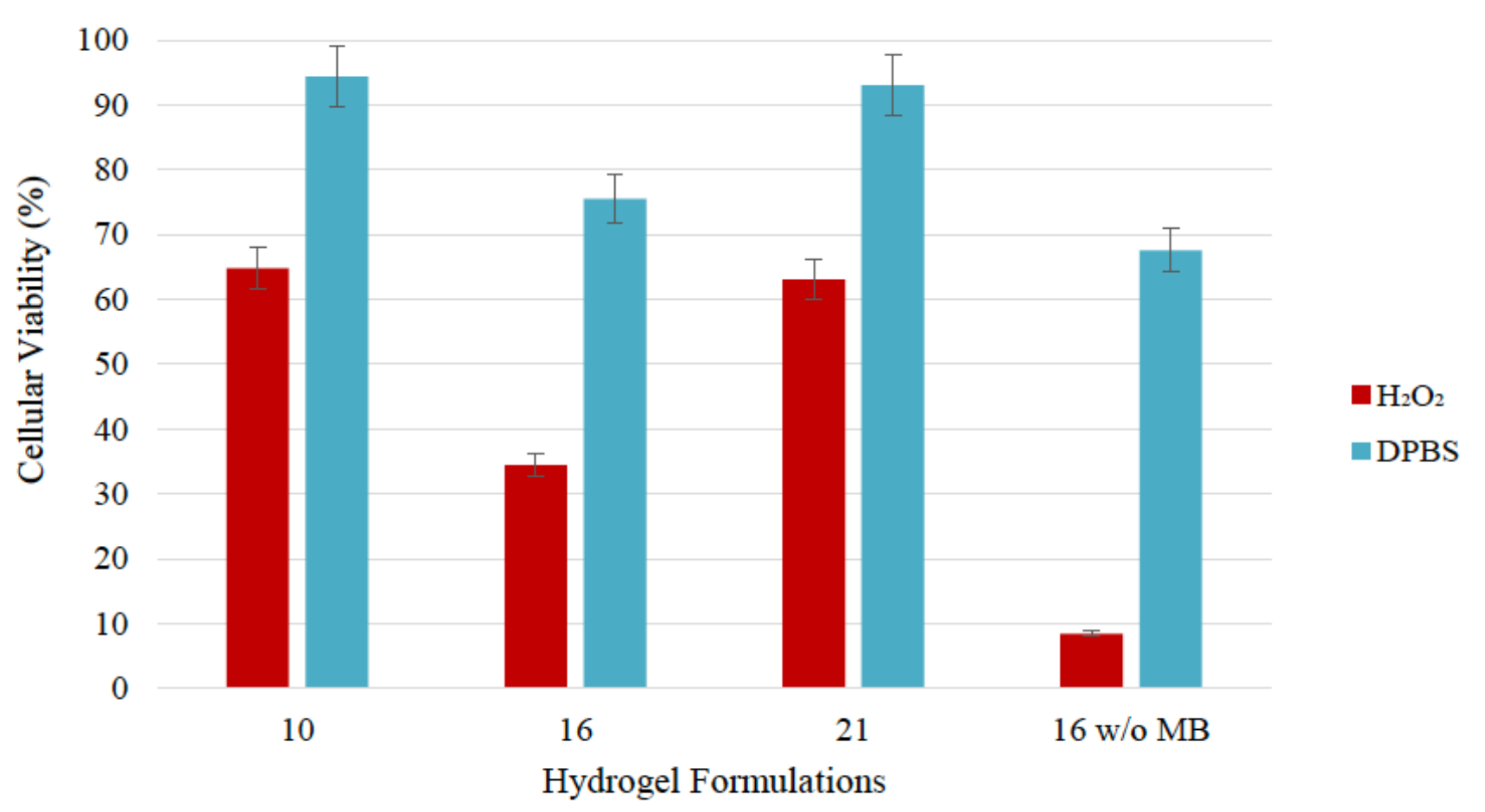

Figure 10

Hydrogel ROS. Cell survival was maintained at over $50 \%$ for formulations 10 and 21 . Cell survival did decrease following exposure to $\mathrm{H} 2 \mathrm{O} 2$. Differences between 10 and 21 were found to not be statistically significant $(p>0.05)$. Differences between formulation 16 with and without MB was found to be statistically significant $(p<0.01)$. 